\title{
Perception and action influences on discrete and reciprocal bimanual coordination
}

\author{
Charles H. Shea $^{1}$ - John J. Buchanan ${ }^{1}$ • Deanna M. Kennedy ${ }^{1}$
}

Published online: 18 August 2015

(C) Psychonomic Society, Inc. 2015

\begin{abstract}
For nearly four decades bimanual coordination, "a prototype of complex motor skills" and apparent "window into the design of the brain," has been intensively studied. Past research has focused on describing and modeling the constraints that allow the production of some coordination patterns while limiting effective performance of other bimanual coordination patterns. More recently researchers have identified a coalition of perception-action constraints that hinder the effective production of bimanual skills. The result has been that given specially designed contexts where one or more of these constraints are minimized, bimanual skills once thought difficult, if not impossible, to effectively produce without very extensive practice can be executed effectively with little or no practice. The challenge is to understand how these contextual constraints interact to allow or inhibit the production of complex bimanual coordination skills. In addition, the factors affecting the stability of bimanual coordination tasks needs to be re-conceptualized in terms of perception-related constraints arising from the environmental context in which performance is conducted and action constraints resident in the neuromotor system.
\end{abstract}

Keywords Bimanual coordination · Perception-action dynamics $\cdot$ Polyrhythm $\cdot$ Coalition of constraints $\cdot$ Optimized displays

Charles H. Shea

CSHEA@TAMU.EDU

1 Department of Health and Kinesiology, Texas A\&M University, College Station, TX 77843-4243, USA

\section{Introduction}

A variety of bimanual movement tasks have been used in experiments investigating the accuracy and stability characteristics of inter-limb coordination. These characteristics have been formally characterized (e.g., Kelso, 1995), extensively investigated (e.g. see Carson, 2005; Fuchs \& Jirsa, 2008; Ivry, Diedrichsen, Spencer, Hazeltine \& Semjen, 2004 for reviews), explained using concepts taken from synergetics (Haken, 1981) and nonlinear dynamical systems, and modeled using nonlinearly coupled limit cycle oscillators (Haken, Kelso, \& Bunz, 1985) perturbed by stochastic forces (Schöner, Haken \& Kelso, 1986). Bimanual coordination experiments "... have revealed quite remarkable temporal constraints between the two hands when they are functioning together" (Kelso \& de Guzman, 1988), such that only a few bimanual movement patterns can be effectively performed without extensive practice. More specifically, the research literature has demonstrated that there are only two inherently stable patterns of bimanual coordination: in-phase and anti-phase (Kelso, 1984) with the in-phase coordination pattern consistently shown to be more stable than the anti-phase pattern (e.g., Kelso, 1981, 1984; Kelso, Scholz, \& Schöner, 1986).

In-phase and anti-phase refer to 1:1 frequency relationships between the movements of the two effectors with $0^{\circ}$ and $180^{\circ}$ phase shift, respectively, with the in-phase pattern often involving the simultaneous activation of homologous muscles of the two limbs and/or mirror movement symmetry. That is, many of the tasks commonly used to investigate bimanual coordination, such as left and right index finger flexionextension (e.g., Riek, Carson, \& Byblow, 1992; Scholz \& Kelso, 1989), forearm pronation-supination (e.g., Carson et al., 1996; Temprado, Zanone, Monno, \& Laurent, 1999), elbow flexion-extension (e.g., Kovacs, Buchanan, \& Shea, 2009a; Spencer \& Irvy, 2007), and circle drawing (e.g., Hiraga, Summers, \& Temprado, 2004; Summers, Maeder, 
Hiraga, \& Alexander, 2008), the in-phase coordination pattern requires the simultaneous activation of homologous muscles. The in-phase pattern is more stable than the anti-phase coordination pattern that requires the activation of nonhomologous muscle groups. However, stable in-phase movements have also been associated with simultaneous activation of non-homologous muscles when visual feedback was manipulated to create perceptual symmetry (e.g., Mechsner, Kerzel, Knoblich, \& Prinz, 2001; Mechsner \& Knoblich, 2004), during multi-joint arm movements (e.g., Buchanan \& Kelso, 1993; Kelso, Buchanan, \& Wallace, 1991), isodirectional movements with non-homologous muscle combinations (e.g., Serrien, Li, Steyvers, Debaere, \& Swinnen, 2001; Serrien \& Swinnen, 1997) and interpersonal coordination (e.g., Oullier, de Guzman, Jantzen, Legarde, \& Kelso, 2008; Schmidt, Carello, \& Turvey, 1990). Regardless of the muscle groups used or the movement of the effectors, the inphase coordination pattern represents a powerful attractor state while the anti-phase pattern is subject to spontaneous phase transitions (loss of stability) to the in-phase pattern when the control parameter (i.e., frequency) is increased (Kelso, 1981,1984, 1995; Kelso, Scholz, \& Schöner, 1986).

This relationship has been illustrated in experiments wherein 1:1 rhythmic movements of the limbs (fingers, wrists, or arms) at a goal relative phase of $30^{\circ}$ to $150^{\circ}$ (e.g., Tuller \& Kelso, 1989; Yamanishi, Kawato, \& Suzuki, 1980; Zanone \& Kelso, 1992), for example, are strongly attracted to in-phase and anti-phase coordination patterns. That is, relative phase patterns other than $0^{\circ}$ and $180^{\circ}$ are not inherently stable and the motor system shows a bias towards what has been labeled the intrinsic dynamics of in-phase and anti-phase coordination (Schöner \& Kelso, 1988). A number of researchers have suggested that the tendency toward in-phase and anti-phase movements of the limbs originates in action constraints of the perception-action system (e.g., Kagerer, Summers, \& Semjen, 2003; Kennerly, Diedrichsen, Hazeltine, Semjen, \& Ivry, 2002; Peper, de Boer, de Poel, \& Beek, 2008), while other researchers suggest that perceptual constraints can play a large role in determining the stability of the bimanual coordination pattern (e.g., Bingham, 2004a,b; Mechsner et al., 2001; Mechsner \& Knoblich 2004). Alternately, a number of studies have favored the hypothesis that a coalition of constraints, ranging from high-level perceptual to lower-level motor, modulates the stability of coordinated behavior (e.g., Amazeen, DaSilva, \& Amazeen, 2008; Carson \& Kelso, 2004; Meesen, Wenderoth, Temprado, Summers, \& Swinnen, 2006; Salesse, Temprado, \& Swinnen, 2005; Temprado et al., 2003; Shea, Kovacs, \& Buchanan, 2009; Swinnen, 2002; Swinnen \& Wenderoth, 2004). In other words, depending on the environmental information available, task requirements, and specific muscle groups utilized multiple constraints may inhibit/disrupt the production of the desired coordination pattern without any one constraint taking precedent at all times.
Research has also demonstrated that similar stability characteristics underlie bimanual multi-frequency coordination (Kelso \& de Guzman, 1988; Peper \& Beek, 1998; Peper, Beek, \& van Wieringen, 1995a). Specifically, research has demonstrated that the $1: 1$ coordination pattern is highly stable, while other ratios (e.g., 1:2, 2:3, 3:5) are significantly more difficult to perform (Fraisse, 1946; see Peper, Beek, \& Van Wieringen, 1995a,b,c, for discussion). As such, individuals tend to transition to stable 1:1 or lower order frequency relationships while performing polyrhythmic coordination patterns (e.g., Peper, Beek, \& van Wieringen, 1995b,c; Treffner $\&$ Turvey, 1993). Thus, when attempting to produce phase or frequency relationships other than 1:1 in-phase, any factor that nudges the movement of one limb toward the pattern of movement of the other limb or perceptual factors related to monitoring the movements of the two limbs could disrupt the already "unstable" coordination pattern and in some cases this results in a phase transition to a more stable (e.g., 1:1 in-phase) coordination pattern (Beek, Peper, \& Stegeman, 1995 for a review).

Although difficult to perform, higher order frequency ratios have been investigated in tapping tasks (e.g., Boonstra, Daffertshofer, Breakspear, \& Beek, 2007; Deutsch, 1983; Klapp, Nelson, \& Jagacinski, 1998; Kurtz \& Lee, 2003), most often with skilled musicians (e.g., Bogacz, 2005; Collier \& Wright, 1995; Peper et al., 1995a,b; Summers, Todd, \& Kim, 1993). Presumably, skilled musicians have developed internal representations of various rhythms that can be used or adapted to produce the goal coordination pattern (see section on visual and auditory models). Higher order frequency ratios have also been investigated in reciprocal motion tasks using handheld pendulums in one or both hands (e.g., Sternad, Turvey, \& Saltzman,1999a,b; Treffner \& Turvey, 1993), crank-turning tasks with the two cranks geared the same (e.g., Boyle, Panzer, \& Shea, 2012; Preilowski, 1972) and geared differently (Mechsner et al., 2001), and have been found to spontaneously emerge in various bimanual tasks, but not maintained for a prolonged period of time (e.g., Buchanan \& Ryu, 2006, 2012; Washburn, Coey, Romero \& Richardson, 2014).

In the sections below we will describe two broad classifications of constraints (perceptual and action) that impact to varying degrees the performance and learning of many bimanual coordination tasks. Perceptual constraints are related to the processing of sensory information available in the performance environment, difficulty associated with attempts to split attentional resources between the movement of the two limbs, and the attentional focus (internal or external) adopted by the performer. Action constraints are related to the contralateral and ipsilateral neural pathways where the activation of one set of muscles can influence the pattern of activation of the homologous and non-homologous muscles in the contralateral limb. Action constraints are often referred to as the product of cortical and sub-cortical neural crosstalk. While the perceptual 
constraints are typically viewed as higher order, more cognitive constraints and motor constraints are often viewed as lower order, there is also evidence for self-organized combinability and exchangeability of these constraints with the coordination dynamics that emerge highly dependent on both the nature of the task, environmental context, and strategy adopted by the performer. Following the introduction to perceptual and action constraints, methods used to minimize the impact of these constraints on reciprocal iso- and multifrequency bimanual coordination tasks, discrete and mixed (discrete and reciprocal) bimanual coordination tasks, bimanual tapping patterns, and finally techniques used to enhance production of circle motion coordination patterns will be described. These methods will then be described both in terms of their immediate impact on performance and longer term impact on learning.

\section{Coalition of constraints}

This section will provide a brief discussion of two classes of constraints that we feel can play powerful roles in the production of a wide range of bimanual coordination tasks and a discussion of factors that may minimize the influence of at least some of these constraints. For the purpose of this review we refer to these constraints as perceptual constraints, referring particularly to visual perception of the limbs and/or enhanced visual displays, and the associated attentional factors present when attempting to monitor metronomes (visual or auditory) and/or the movement of the two limbs. Attentional factors refer particularly to conditions which may distract the performer from more salient movement information and/or testing conditions that require attention to be split between effectors or stimuli and/or result in changes from the optimal attentional focus. Action constraints arise from contralateral and ipsilateral neural pathways that activate the muscles used to produce the coordination pattern. One type of action constraint is neural crosstalk whereby some portion of the force commands for one limb is diverted to the other limb. This has the potential to periodically perturb the movement of the contralateral limb when the two limbs are moving at different phase or frequency relationships or alternatively in the case of in-phase movement serve to stabilize the movement pattern.

\section{Perceptual constraints}

The nature of non-linear coupling among the component oscillators that were represented in abstract mathematical terms in the HKB model (Haken et al., 1985; Schöner et al., 1986; Haken, Peper, Beek, \& Daffertshofer, 1996) has also been repeatedly linked to the perceptual information available. Research has revealed a pivotal role for proprioception
(Baldissera, Cavaliari, Marini, \& Tassone, 1991; Ridderikhoff, Peper \& Beek, 2005) and tactile input (Aschersleben \& Prinz, 1995; Buchanan \& Ryu, 2005; Kelso, Fink, DeLaplain, \& Carson, 2001) in stabilizing in-phase and anti-phase coordination. Clues as to the importance of visual perceptual information to bimanual coordination have also been found in interpersonal coordination tasks (Schmidt et al.,1990), where two people coordinate their movements, and unimanual tracking tasks (Wilson, Collins, \& Bingham, 2005a,b), whereby performers coordinate their movements to an external signal, which also exhibit stable coordination patterns at $0^{\circ}$ and $180^{\circ}$ relative phase. In the interpersonal coordination paradigms a direct neuromuscular connection between the components is not present, suggesting coupling occurs at a perceptual level. Bingham and colleagues (e.g., 2004a,b; Bingham, Schmidt, \& Zaal, 1999; Wilson, Collins, \& Bingham, 2005a,b) have also argued that bimanual coordination can be limited by the performer's ability to detect a given relative phase pattern through visual perception. That is, if a participant rates the movement of two horizontally moving dots in a display as uncoordinated and cannot distinguish the amount of variability in the pattern, then it is unlikely they will be able to produce a bimanual coordination pattern consistent with the display. Using this logic, the reason for poor performance in bimanual tasks when using visually based environmental information to specify the desired relative phase is that participants are unable to detect their errors, and thus are unable to initiate effective corrections. The tacit assumption of this argument is that if salient perceptual information is provided which facilitates pattern detection then error detection (and correction) will follow. The extension of this argument is that the end result will be increasingly stable performance achieved through practice. The work of Bingham and colleagues establishes a link between visual perception and the stability of symmetric and asymmetric coordination patterns, but they did not directly test the role that such perceptual processes actually play or can play in the performance or learning of bimanual coordination patterns. Similarly, Mechsner et al. (2001) provided evidence that under specific postural manipulations coordinated bimanual movements were organized in terms of visual symmetry and not motoric symmetry as previous work suggested (see Riek et al., 1992 for alternative findings). That is, Mechsner et al. have shown that a bimanual coordination pattern in which the simultaneous activation of non-homologous muscles is required can be performed as stably as a coordination pattern requiring simultaneous activation of homologous muscles, provided there is visual symmetry of movement direction. However, Mechsner's view whereby coordinated movements are purely perceptual-cognitive/psychological in nature (Mechsner \& Knoblich, 2004) has been intensively questioned by many scientists (e.g., commentaries on Mechsner and Knoblich, 2004, "A psychological approach to human voluntary movements," and responses of the author). 
One perceptual manipulation used in an attempt to overcome the difficulty participants experience in conceptualizing the bimanual task and in detecting and correcting coordination errors involves the use of performance goals presented through Lissajous portraits and concurrent Lissajous displays. Terminal Lissajous templates and feedback have been used with some success in bimanual experiments requiring individuals to learn novel 1:1 coordination patterns with various phase lags (e.g., Hurley \& Lee, 2006; Lee, Swinnen, \& Verschueren, 1995; Swinnen, Dounskaia, Verschueren, Serrien, 1995; Swinnen, et al., 1997b; Swinnen, Verschueren et al., 1998). For example, Swinnen, Lee et al. (1997b) had participants practice a 1:1 bimanual coordination pattern with a $90^{\circ}$ phase offset for 3 days ( 50 trials per day) under various feedback conditions. Most relevant to the present discussion were conditions with and without online Lissajous feedback. A Lissajous plot integrates the position of the two limbs into a single point. For example, the movement of one limb would move the cursor horizontally with extension of the limb moving the cursor to the right and flexion moving the cursor to the left while the motion of the other limb moves the cursor vertically (flexion-down, extension-up). Thus, a 1:1 movement pattern with a $90^{\circ}$ phase offset would result in circular movement of the cursor. Swinnen, Lee et al. (1997b) found enhanced coordination performance and decreased performance variability during acquisition for the group provided terminal Lissajous feedback relative to the group without the Lissajous information. Performances on delayed retention and transfer tests without Lissajous displays were also enhanced following acquisition with Lissajous feedback.

It should be noted that a Lissajous plot as a source of perceptual information may also serve to reduce attentional processing demands to a more manageable level because the participant's attention does not have to be split between the movements of the two limbs. Instead, attentional resources may be directed towards the integrated representation of the two limbs in the Lissajous plot. That is, coordination errors may be more easily detected and thereby corrected, especially when a goal movement pattern template is provided in the Lissajous display. However, if participants do split attention between direct vision of the limb movements and the transformed movement of the limbs in the Lissajous display, the information could be conflicting because the planes of motion are different. In addition, participants have a strong tendency to attempt to actively control limb motion when the limbs are visible, which in a relatively large number of experiments has been shown to have a negative impact on performance (see Wulf, 2007 for review).

Research has also shown that cognitive processes, such as intention (Scholz \& Kelso, 1990), strategy selection (Kelso, DelColle \& Schöner, 1990), and instruction (Fowler \& Turvey, 1978) can influence the stability of bimanual and unimanual coordination patterns. Recent theorizing has attempted to link neural areas such as the anterior cingulate cortex to cognitive constraints that may impact the "functional representations" of muscles underlying sensorimotor coordination in general (Carson \& Kelso, 2004). For example, when trained musicians (pianists) performed a familiar piece, increased activation was found in the SMA whereas when they performed unfamiliar pieces (increased attentional demands) increased activation was found in the pre-SMA (Sergent, 1993). Thus, some researchers view the basis for the functional asymmetry as mediated by perceptual factors related to attention (Peters, 1989, 1994; Kinsbourne, 1970). Indeed, the role of attention in bimanual movements has received a good deal of experimental investigation (e.g., Hiraga et al., 2004; Summers et al., 2008). However, there are also views arguing that while attentional factors undoubtedly play a role, they are not the basis for the functional asymmetry (Carson, 1989; Allen, 1983; Amazeen, Amazeen, Treffner \& Turvey, 1997), but rather attention and handedness are related through their mutual effects on the bimanual coordination dynamics (de Poel, Peper, \& Beek, 2008; Treffner \& Turvey, 1995, 1996). Further, Temprado, Zanone, Monno, and Laurent (1999) have demonstrated that the stability of the intrinsic dynamics (i.e., in-phase and anti-phase) as well as the difference between them can vary based on the attentional priority given to the coordination task. When attention to the bimanual task was somehow released (i.e., shared attention in a dualtask condition or focus on the secondary task) both intrinsic patterns showed a consistent increase in variability with higher increases for the anti-phase compared with the inphase pattern. Conversely, when attention was directed to the bimanual task, variability of both patterns decreased with a stronger effect on the anti-phase pattern. Furthermore, these effects, observed at the level of the coordination pattern, were also reflected at the component level (individual limbs).

In terms of attentional factors influencing multifrequency coordination patterns (i.e., 2:3), Peters and Schwartz (1989) have shown that attention directed to the slower moving limb typically leads to larger performance decrements than attention directed to the faster moving limb. Other research has demonstrated that bimanual coordination is also constrained by whether the movements involve the activation of homologous muscles and/or mirror symmetry movements, and that abstract directional codes through practice become part of the memory representation for bimanual movements and can constrain transfer more so than muscle pairs (Temprado \& Swinnen, 2005). Swinnen and Wenderoth (2004) have maintained that attentional factors interact with the motoric aspect of the coupling that drives the phase attraction towards in-phase and anti-phase coordination (Swinnen \& Wenderoth, 2004).

Recently, two relatively specific attentional factors have been shown to provide strong constraints on bimanual 
coordination (Kovacs et al. 2009a; Kovacs, Buchanan, \& Shea, 2009b); one factor involves vision of the limbs when alternative forms of concurrent feedback are provided (e.g., Lissajous plots) and the second factor is related to the use of visual and auditory metronomes used to pace cycle frequency of the limbs. Visually monitoring the movement of the two limbs in bimanual coordination appears, at first glance, to be a very natural way to facilitate the production of bimanual coordination patterns. However, for a number of reasons visual monitoring of the limbs may actually prove detrimental to coordination. A growing literature suggests that monitoring limb movement encourages explicit control of the limbs, and this may actually interfere with more implicit control processes. Research on attentional focus has shown that participants tend to relinquish conscious control when limb motion is hidden from view and/or when the participant's attention is directed to other salient information (see Wulf, 2007 for review). For example, McNevin, Shea, and Wulf (2003), found an improvement in stabilometer performance when participants' attentional focus was directed away from their feet and legs. Frequency analysis (FFT) of the platform's motion indicated an overall increase in mean power frequency of the platform's motion with an overall decrease in amplitude when attention was directed away from the feet and legs relative to when participants were instructed to monitor their lower limb movements. The authors argued that focusing attention on the limbs compromised (or constrained) the normal regulatory processes involved in balance. McNevin et al. referred to this as the "constrained action hypothesis" because it appeared that attention directed to the limbs increased the likelihood that participants exerted conscious control of the limbs inhibiting more efficient control processes. As suggested by the stabilometer study, providing vision of the limbs during a bimanual coordination task may well increase the likelihood that participants attempt to consciously intervene in the control of one or both limbs to the detriment of bimanual performance.

A second attentional factor involves the use of visual and auditory metronomes. Note that metronomes have not only been used to pace bimanual coordination at a particular frequency, but have also been used to provide a way of directing the participant to the goal coordination pattern. In Kelso and Zanone (2002), for example, "participants were instructed to produce the required relative phase specified by the visual metronome" by synchronization of the reversal of the right limb with the onset of the right LED and reversal of the left limb with the onset of the left LED. If participants were able to do this effectively, they would achieve the goal phase offset and the goal frequency. However, to do this attention must be utilized to compare the movements of the limbs with the timing information provided by the metronomes. Thus, attentional load may increase when metronomes are used and this load may be further increased when the cycle frequency is increased.

\section{Action constraints}

The tendency for participants to transition from asymmetric coordination patterns to in-phase movements of the fingers or limbs has been thought to be due, at least in part, to biases originating in the action component of the perception-action system (e.g., Kagerer et al., 2002, 2003; Peper et al., 2008). For example, the concept of neural crosstalk has been used to explain the findings of stability differences and phase transitions in various bimanual coordination patterns based on interactions in the forward command streams in the highly interconnected and redundant organization of the nervous system (for review, see Swinnen, 2002). Neural crosstalk has been defined as a mirror image command sent to the homologous muscles of the contralateral limb (e.g., Cattaert, Semjen, \& Summers, 1999; Swinnen, Lee et al., 1997b). According to the crosstalk model, two independent motor plans exist for each limb and some fraction of the force commands for one limb is diverted to the other limb (Cattaert et al., 1999). It has been proposed that the movements produced by the symmetric activation of homologous muscles are stabilized when the contralateral and ipsilateral signals are integrated, while movements produced by the activation of nonhomologous muscles or asymmetric activation suffer from ongoing interference due to conflicting information and partial intermingling of signals controlling the two arms (e.g., Kagerer et al., 2003; Kennedy, Boyle, Rhee, \& Shea, 2015a; Marteniuk, MacKenzie, \& Baba, 1984).

Researchers have demonstrated that the temporal control of discrete and continuous bimanual movements have distinct underlying neural mechanisms (Robertson \& Murre, 1999). The temporal control of discrete movements, such as finger tapping, requires the specification of a series of discrete signals related to the endpoint of movements. In contrast, the temporal control of reciprocal movements requires the signal to specify a continuous spatial-temporal relationship between components (limbs). It appears that (especially for reciprocal bimanual movements), there is no single location in the brain responsible for the control of the spatial-temporal patterns of bimanual coordination; rather a distributed neural network is involved (Debaere et al., 2001). Furthermore, the brain areas involved/activated also differ depending on the types of coordination patterns performed. For example, activation levels in the SMA, S1, M1, CMA, and PM were higher when an antiphase coordination pattern was performed compared to when an in-phase pattern was performed (Jancke et al., 2000; Stephan et al., 1999). Also, Cardoso de Oliviera, Gribova, Duchin, Bergman and Vaadia (2001) have shown that during in-phase coordination the correlations between interhemispheric motor cortical areas (the motor areas activated simultaneously in both hemispheres) are much stronger than during anti-phase coordination. As Swinnen (2002) noted, this correlated activity between hemispheres could provide the neural 
basis of crosstalk between limbs, as observed at the behavioral level. In other words, neural crosstalk occurs during bimanual coordination when both hemispheres send signals to the contralateral side of the body via the crossed cortico-spinal pathways, yet also simultaneously send the same signal to the ipsilateral side of the body via uncrossed cortico-spinal pathways.

When in-phase coordination is performed the signals coming to the muscles of one limb from both contralateral and ipsilateral pathways are congruent (homologous muscles are activated), and thus not conflicting. However, when an antiphase pattern is performed (requiring the simultaneous activation of non-homologous muscles), each limb (depending on the timing) will receive conflicting signals from the contralateral and ipsilateral hemisphere (Cattaert et al., 1999; Kagerer et al., 2003; Kennerley et al., 2002). Interestingly, the likelihood of interference resulting from non-congruent signals appears to increase as cycle frequency and/or force requirements increase and may even delay the onset of force commands (Kennedy, Wang, Shea, 2013a,b). Moreover, the notion of neural cross-talk combined with the identified activation areas might give us an indication why musicians are able to perform certain difficult coordination patterns much better than nonmusicians. Jancke et al. (2000) noted that cortical areas activated as well as activation levels change as a function of practice with more activation in more extended areas early in practice. Pianists show lower degrees of activation in primary and secondary motor areas than non-musicians. Consequently, lower activation levels might yield less conflicting signals via uncrossed cortico-spinal pathways for musicians. Some research has shown that with the loss of somatosensory feedback there is a decrease in the stability of anti-phase coordination, however, this loss does not keep the system from establishing and maintaining symmetric and asymmetric bimanual circle tracing patterns (Spencer, Ivry, Cattaert, \& Semjen, 2005). Thus, one contribution to the coupling and stability characteristics of bimanual coordination clearly resides in forward commands and the interactions that arise from those commands as the result of shared neural pathways (Ridderikhoff et al., 2005). It should be noted that constraints related to crossed and uncrossed neural pathways should affect to varying extents all iso- and poly-rhythmic bimanual coordination patterns except the in-phase 1:1 coordination pattern.

Kennedy, Boyle, Wang, and Shea (2015b) designed a series of experiments to determine the level of cooperation or interference observed from the forces generated in one limb on the forces produced by the contralateral limb when one or both limbs were producing a constant force (Experiment 1), one limb was producing a dynamic force while the other limb was producing a constant force (Experiment 2), and both limbs were producing dynamic force patterns (Experiment 3). The results for both Experiments 1 and 2 showed relatively strong positive time series cross-correlations between the left and right limb forces indicating that an increase or decrease in the force generated by one limb resulted in corresponding changes in the force produced by the homologous muscles of the contralateral limb. Experiment 3 required participants to coordinate in-phase 1:1 and 1:2 bimanual force patterns. This experiment will be discussed in more detail in the section on multi-frequency bimanual coordination; however, it is important to note that relatively small but identifiable influences of right limb forces on the left limb force time series were also observed in the 1:2 task that were not evident in the 1:1 inphase task. The results of all three experiments provided evidence to support the notion that action constraints related to crossed and uncrossed neural pathways influence bimanual coordination. It is important to note, however, that these perturbations in the force time series were only minimally disruptive to the management of the coordination pattern while Lissajous feedback was provided. Thus, it appears that the Lissajous feedback did not eliminate the action constraints, yet its use did minimize their impact on the overall coordination pattern.

It should also be noted that the influences of action constraints appear to be highly dependent on the task requirements and the specific limb/muscles that are utilized. The use of ipsilateral muscle groups, for example elbow and wrist of the same limb, would be impacted differently than the use of homologous muscles of the contralateral limbs. Similarly, tasks that required increased forces for one limb may result in larger influences on forces produced by the contralateral limb movement than those produced in near frictionless environments (e.g., movements of levers).

\section{Minimizing perceptual constraints while managing action constraints}

One technique that appears to have great potential to reduce perceptual constraints involves the use of Lissajous displays. Lissajous displays integrate the position of the two limbs into a single point in one plane. That is, the movement of two limbs is displayed as a single point (cursor) where movement of one limb moves the cursor to the left or right while the movement of the other limb moves the cursor up and down. It should be noted that a Lissajous display as a source of perceptual information may reduce perceptual demands because the participant's attention does not have to be split between the movements of the two limbs in order to determine the relationship between the limbs. Instead, perceptual resources may be directed towards the integrated representation of the two limbs in the Lissajous display. However, Lissajous displays are not the only method that has been shown to be effective in reducing the perceptual constraints associated with bimanual coordination. 
In the following subsections a variety of bimanual tasks and integrated displays will be discussed in terms of the impact of integrated displays on bimanual coordination. We start with the influence of Lissajous displays on 1:1 bimanual coordination with various phase shifts, the production of a variety of multi-frequency bimanual coordination patterns, and discrete and combined discrete and reciprocal bimanual coordination tasks. Next we describe the effectiveness of providing online error information in the production of bimanual circling movements and, lastly, we describe the use of integrated visual and auditory models on the production of bimanual tapping rhythms.

\section{1:1 bimanual coordination}

A series of bimanual coordination experiments (Kovacs et al., 2009a,b) were conducted in an attempt to determine if bimanual 1:1 coordination patterns with various phase shifts are more stable than typically demonstrated when (1) salient, integrated feedback is provided, (2) attention demands are reduced, and (3) attempts to consciously coordinate the limbs are not encouraged ( see McNevin et al., 2003 for a discussion of external and internal foci of attention). These experiments were designed in an attempt to minimize and document at least some of the perceptual constraints thought to influence performance in many bimanual coordination experiments.

Two experiments by Kovacs et al. (2009a,b; Also see Kovacs \& Shea, 2010) using 1:1 bimanual coordination patterns with various phase shifts manipulated the presence of Lissajous feedback, the use of metronomes, and vision of the limbs. Kovacs et al. (2009a) asked participants to produce a $90^{\circ}$ relative phase bimanual coordination pattern (elbow flexion and extension). All participants received concurrent Lissajous feedback either with or without an auditory metronome $(1 \mathrm{~Hz})$; vision of the limbs was occluded for both groups. Participants not provided a metronome were simply encouraged to increase their cycling frequency on subsequent trials if their frequency fell below the $1 \mathrm{~Hz}$ goal. Performance after only 5 min of practice indicated quite remarkable coordination for the no-metronome condition (relative phase variability and absolute error in relative phase $<10^{\circ}$ ) (Fig. 1a) compared to the metronome condition (relative phase variability and absolute error in relative phase $\sim 30^{\circ}$ ) (Fig. 1b). These data are important because they demonstrate the strong negative impact of utilizing a metronome to pace bimanual coordination tasks. The results are also helpful in explaining why participants provided Lissajous feedback and a metronome to pace their movements in earlier experiments, yet did not have vision of their limbs because testing was conducted in a functional magnetic resonance imaging (fMRI) scanner (e.g., Debaere et al., 2001; Debaere, Wenderoth, Sunaert, Van Hecke, \& Swinnen, 2003; Puttemans, Wenderoth, \& Swinnen, 2005) or had vision of their limbs occluded for some other reason (e.g., Summer, Davis, \& Byblow, 2002), did not perform particularly well even though they were provided substantially more practice than in the Kovacs et al. (2009a) experiment. Note that the performance of the various groups at the end of the 5-min practice period were under the same conditions that they had been practicing under.

The second experiment (Kovacs et al., 2009b) addressed the question: Can participants produce with low error and variability a wide range of relative phase patterns (between $0^{\circ}$ and $180^{\circ}$ in $30^{\circ}$ increments) using a protocol similar to that of Yamanishi et al. (1980) and Zanone and Kelso (1992) but with Lissajous feedback provided, vision of the limbs occluded, and when metronomes are not used. Participants in one group were provided concurrent Lissajous feedback, while participants in another group where provided visual metronomes to guide the left- and right-limb cycle frequency. The task was to flex and extend the two limbs in order to move the Lissajous cursor in the pattern depicted by a template in the display (Fig. 2a,b). Note that the template changed depending on the task for that trial $\left(0^{\circ}\right.$ to $180^{\circ}$ in $30^{\circ}$ increments $)$. All participants completed three practice blocks and one test block. The metronome group produced patterns of relative phase error and variability similar to those observed in previous experiments. However, in the Lissajous group these measures were substantially reduced to levels typically observed only after multiple days of practice, and then only for the relative phase pattern that was practiced (Fig. 2c-i). Importantly, relative phase variability was less than $10^{\circ}$ for all tasks except in-phase $\left(0^{\circ}\right)$ where relative phase variability was reduced. These findings again were consistent with the hypothesis that Lissajous feedback provides salient information that allows participants to quickly "tune-in" the necessary motor commands to produce a desired relative phase relationship. We and others (e.g., Mechsner et al., 2001) have described the process of adjusting the cycle frequency as "tuning in" the goal bimanual coordination pattern because it appears that participants increase and/or decrease the cycle frequency of the two limbs until they find the movement pattern that best matches the goal template provided in the Lissajous display. It is important to note that this experiment again provides evidence that the use of metronomes to guide and pace bimanual movements may actually be detrimental to the performance of the goal coordination pattern. However, these techniques do not appear to result in the development of an internal representation of the task that can be used when and if the Lissajous display is withheld. That is, when the Lissajous display is removed performance deteriorates.

\section{Multi-frequency bimanual coordination}

Kovacs, Buchanan, and Shea (2010a,b; also see Boyle et al., 2012) designed experiments in an attempt to demonstrate that difficult multi-frequency coordination between the limbs can 

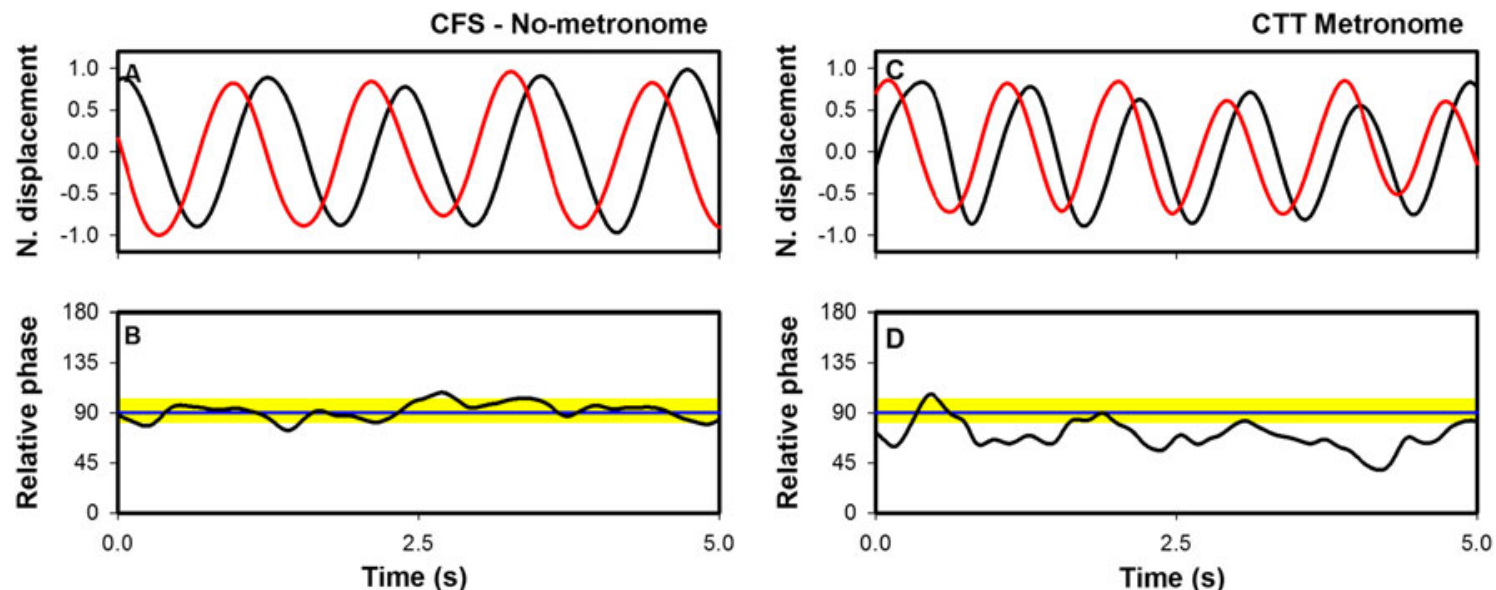

Fig. 1 Actual left and right limb movements and resulting relative phase for a participant in the no-metronome condition $(\mathbf{A}, \mathbf{B})$ and a participant in the metronome conditions (C,D) (from Kovacs et al., 2009b)

be performed more effectively than typically demonstrated when (1) salient, integrated feedback is provided, (2) attention demands are reduced, and (3) attempts to consciously coordinate the limbs are not encouraged. Kovacs et al. (2010a)
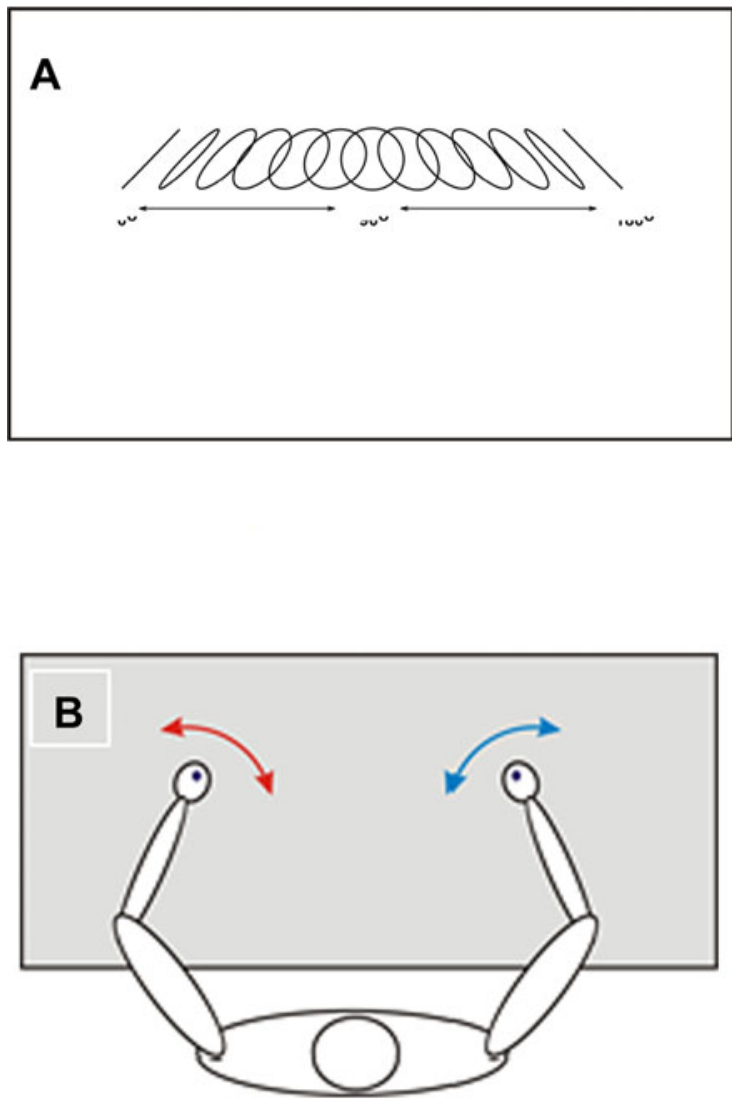

C

D

$\mathbf{E}$

$\mathbf{F}$

G

H

Fig. 2 Example of the Lissajous displays (A) and participant set-up (B). Examples of normalized left and right limb displacements (left) and resulting Lissajous plots (right) for the various scanning conditions $(\mathbf{C}$ - investigated 2:1 and 3:2 bimanual coordination patterns and Kovacs et al. (2010b) investigated 5:3 and 4:3 bimanual coordination patterns. Note that 5:3 and 4:3 bimanual coordination patterns were thought to be significantly more difficult

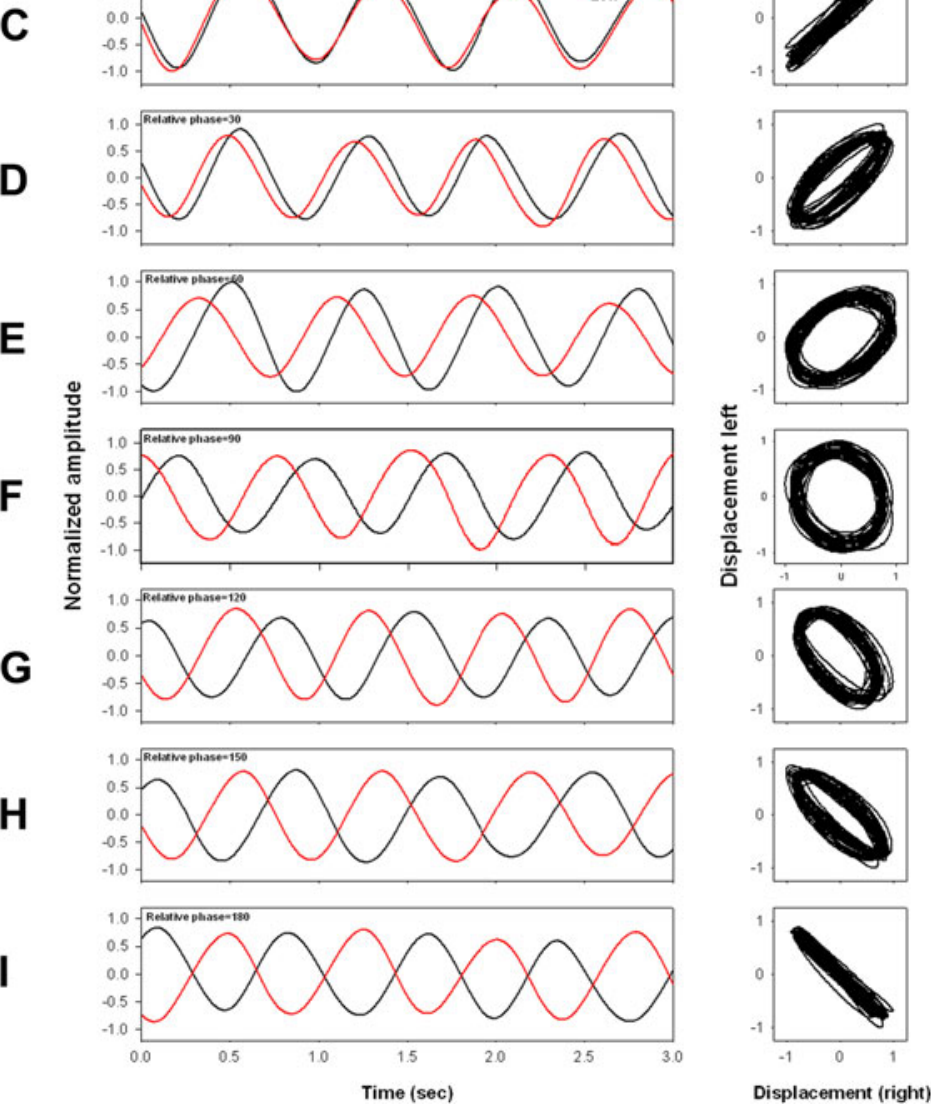

I) are presented. Note that the sequence of Lissajous templates displayed was presented one at a time (from Kovacs et al., 2009a) 
than $2: 1$ and $3: 2$ coordination patterns. Indeed, as noted earlier, each of these patterns were thought to be extremely difficult to perform effectively without extensive practice.

In Experiment 1 of Kovacs et al. (2010a) the goal was a 2:1 harmonic ratio (Fig. 3a,b), and in Experiment 2 the goal was a more difficult $3: 2$ polyrhythmic ratio (Fig. 3c, d). In both experiments participants were asked to produce the required frequency ratio by continuously flexing and extending the elbows in the horizontal motion plane. One group of participants was permitted vision of their limbs and provided Lissajous feedback (uncovered limb/ Lissajous group). Two additional groups performed the task while the limbs were blocked from view. One group was provided Lissajous feedback and the other group was not provided Lissajous feedback. Comparison of the uncovered and covered limb Lissajous groups allows the determination of the effect of vision of the limbs while using Lissajous feedback. The authors hypothesized that the uncovered limbs/Lissajous condition would encourage participants to attend to the motion of the two limbs and the relationship of these motions to the cursor on the screen, whereas attention in the covered limb/Lissajous condition would be directed to the motion of the cursor in the Lissajous display. The authors predicted the difference in attention allocation would result in a significantly more stable production of the multi-frequency ratios by the covered limb/Lissajous group following minimal practice $(4.5 \mathrm{~min}$ ) in comparison to the uncovered limb/ Lissajous group. Comparison of the performance of the covered limb/Lissajous and covered limb/no Lissajous conditions allowed the determination of the influence of Lissajous displays.
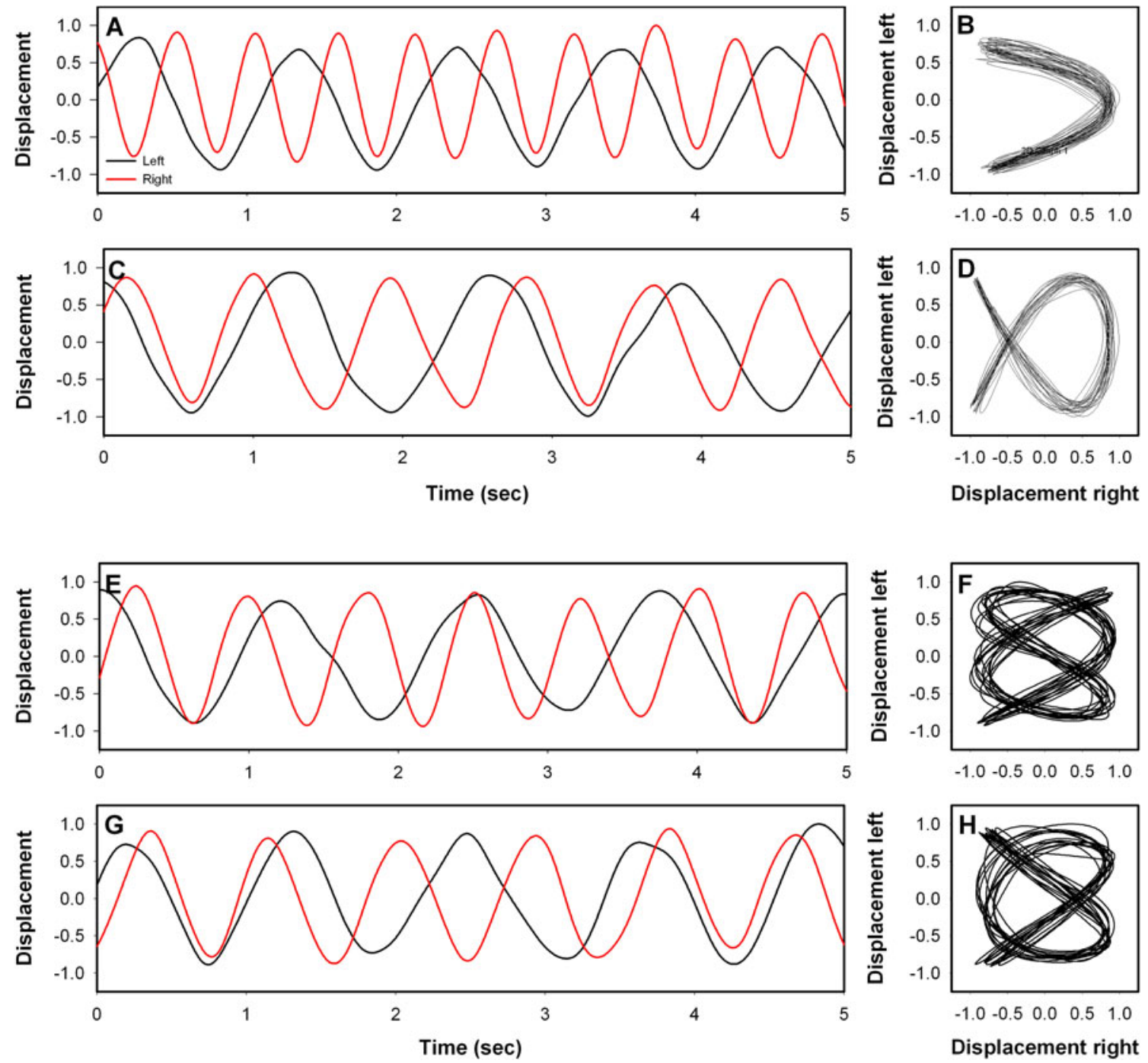

Fig. 3 Examples of the normalized left and right limb displacement and resulting Lissajous plots for a 2:1 (A,B) and 3:2 (C,D) bimanual ratio in the Lissajous condition (Kovacs et al., 2010a) and examples of the normalized left and right limb displacement and resulting Lissajous plots for

a $5: 3(\mathbf{E}, \mathbf{F})$ and 4:3 $(\mathbf{G}, \mathbf{H})$ bimanual ratios in the Lissajous condition (Kovacs et al., 2010b). Note that following practice with the 5:3 ratio the participant was tested on the 4:3 ratio with no prior practice on that coordination pattern (from Kovacs et al., 2010a,b) 
In both experiments participants were not permitted to view their limbs, yet were provided with vision of the cursor and goal template presented in a Lissajous plot (covered limb/ Lissajous groups), were able to effectively perform the 2:1 harmonic pattern and the 3:2 polyrhythmic pattern of bimanual coordination with less than 5 min of practice. Both multifrequency patterns were maintained not only at discrete points (e.g., beginning and ending of the rhythm cycle), consistent with discrete tapping work (e.g., Bogacz, 2005; Collier \& Wright, 1995; Peper et al., 1995a,b), but also continuously throughout the movement. When producing the 2:1 and 3:2 bimanual coordination tasks both the left and right limb motions were harmonic. However, when vision of the limbs was permitted and Lissajous information was provided (uncovered limb/Lissajous groups), the production of the 2:1 coordination pattern was disrupted and the harmonic nature of the left-arm's motion was reduced compared to when a Lissajous display and goal template were provided and the limbs were covered. Indeed, the disruptions resulting from vision of the limbs while using Lissajous displays were larger in the 3:2 task than in the 2:1 task. When the limbs were covered and no Lissajous information was provided bimanual performance was quite poor in both the 2:1 and 3:2 tasks. Apparently, covering the limbs was necessary for the system to more fully exploit the perceptual information provided in the Lissajous display.

The purpose of the Kovacs et al. (2010b) experiment was to extend these results to even more difficult polyrhythmic coordination patterns and to determine if participants could after practice with one coordination pattern $(5: 3)$ rescale their responding to a new, equally difficult coordination pattern (4:3). The data indicated that participants could effectively perform a 5:3 coordination pattern after $10 \mathrm{~min}$ of practice, and then on the first attempt adjust their movements so as to effectively produce the unpracticed $4: 3$ coordination pattern (Fig. 3e-h). Note that the 5:3 and 4:3 coordination patterns were thought to be virtually impossible to achieve in reciprocal motion tasks even after extensive practice. The level of bimanual performance attained in this experiment was remarkable, especially when compared to the performance levels achieved in previous research using Lissajous feedback wherein performers required several days of practice to achieve similar levels of performance on a relatively simple (at least in comparison) $1: 1$ with $90^{\circ}$ phase lag (e.g., Hurley \& Lee, 2006; Lee et al., 1995; Swinnen et al., 1998; Swinnen, et al., 1997b) and 2:1 ratios (Swinnen, et al., 1997a; Summers et al., 2002). Note also that participants were asked to perform the $4: 3$ coordination pattern after practicing the 5:3. However, no practice was provided on the $4: 3$ prior to the test trial and no instructions or warning was provided to the participants that the task requirements were going to change. The only change in the protocol was that the Lissajous template was changed. Remarkably, the participants were able to effectively produce the new coordination pattern with no previous practice on that specific task. Note that participants from the previous experiments were tested under the same conditions that they had practiced under during the acquisition trials.

This line of research was extended to include the coordination of multi-frequency patterns of force (Kennedy et al., 2015a,b). The authors argued that by using Lissajous displays to perform a 1:2 bimanual force coordination pattern with a goal template that many of the perceptual constraints would be minimized, allowing the detection of action constraints that may result from neural crosstalk. Isometric force was used in the hope that the increases in force requirement will allow the subtle impact of neural crosstalk to be more easily detected. A 1:1 pattern of force was used as a control. The task was to rhythmically produce a pattern of isometric forces on a left-side force transducer with the left arm that was coordinated with the pattern of isometric forces produced on a right-sided force transducer with the right limb. Similar to earlier investigations using movements produced in near frictionless environments, the results indicated very effective performance of both bimanual force coordination patterns. However, identifiable and consistent distortions in the force produced by the left limb were observed when the right limb was initiating or releasing a force pulse during the 1:2 task that were not evident in the $1: 1$ task.

In another investigation, Kennedy and colleagues (2015a,b) compared participants' ability to coordinate bimanual multi-frequency patterns of isometric forces using homologous or non-homologous muscles. Lissajous feedback was again provided to guide performance. The purpose was to determine whether the activation of homologous and nonhomologous muscles resulted in different patterns of distortions in the left limb forces that are related to the forces produced by the right limb. The results indicated that participants were able to perform the 1:2 multi-frequency pattern with both homologous and non-homologous muscles. However, distinct but consistent distortions in the left limb force traces were observed in the two tasks. In the homologous task the interference occurred in the left limb at the point of right limb muscle activation and the release of force. In the nonhomologous task the interference in the left limb force occurred only when the right limb was releasing force. Taken together, the results of these force tasks provide further evidence for the robust utility of perceptual displays in facilitating multi-frequency bimanual coordination patterns (Boyles et al., 2012; Kennedy et al., 2015a,b; Kovacs et al., 2010a,b; Kovacs \& Shea, 2011; Mechsner et al. 2001).

\section{Discrete and discrete-reciprocal bimanual coordination}

Papers by Kelso, Southard, and Goodman (1979a,b) published over 35 years ago and now classic, compared 
participants' reaction time (RT) and movement time (MT) in producing single limb movements of the left or right hand/ finger from a home position to targets with the simultaneous movement of the two hands/fingers to separate targets. To start a trial in the one-handed conditions participants depressed the left home key with the left index finger for left-handed movements or the right home key with the right index finger for right-handed movements. Upon hearing the auditory start signal the participant moved as quickly as possible to the designated target on that side depressing the key with the respective index finger. In the two-handed conditions participants depressed both left and right home keys with the respective index finger and moved each hand/arm to the target on the respective side upon hearing the start signal. The movement time results were consistent with Fitts' law for single limb aiming movements and bimanual movements to targets with the same amplitude and target width (same ID).

In the mixed conditions the easy (low ID) movements were performed more slowly than would be expected given the low ID and single limb performance, while the difficult (high ID) movements were performed consistent with the high ID and similar to when single limb movements were required. In the mixed condition the low and high ID movements resulted in different velocities, but the velocity profiles (e.g., time of peak velocity) were almost perfectly synchronous (also see Kelso, Putnam, \& Goodman, 1983). In Experiments 2 and 3 the targets were placed so that movements were toward the midline or away from the body, yet the results were remarkably similar to that from Experiment 1 (also see Heuer \& Klein, 2006; Marteniuk et al., 1984; Spijkers, Tachmatzidis, Debus, Fischer, \& Kausche, 1994). Taken together, these findings were interpreted by Kelso et al. as indicating that the "limbs are constrained to act as a single unit" with the more difficult task appearing to determine the time course of both limb movements. This finding not only demonstrated an important exception to Fitts' law, but also served to focus increased attention on the constraints that impose limitations on a wide range of bimanual coordination tasks. Interestingly, RT was also influenced by whether one or two movements were executed and whether the two movements were the same or different. The finding that RT increased when two movements were required compared to when one movement was required was not surprising, yet was interesting because RT increased in the mixed condition when the two movements were different compared to when the two movements had the same amplitude and target width requirements. This was interesting because the participants produced the two movements in nearly the same movement time, but RT was longer. This suggests that some additional planning/programming was required to move the two limbs to different amplitudes in the same movement time.

Similarly, Diedrichsen, Hazeltine, Kennerley, and Ivry (2001; also see Ivry, Diedrichsen, Spencer, Hazeltine, \&
Semjen, 2005, for review) determined RT for unimanual and bimanual reaching movements. More specifically, they examined the source of bimanual interference by comparing RT under various reaching conditions involving symmetric bimanual movements with conditions involving asymmetric bimanual movements of different amplitudes (Experiment 1) or direction (Experiment 2). The target movements were cued either symbolically with letters or directly by the onset of the target location. The results indicated longer RTs for asymmetric movements than for symmetric movements during trials that were cued symbolically. However, RTs were the same for symmetric and asymmetric trials that were cued directly. The researcher attributed these findings to interference during the processing of the information rather than interference at the motor programming stage. In a follow-up investigation, Diedrichsen and colleagues (2003) conducted an additional series of experiments to identify the locus of the interference during the preparation of bimanual reaching movements. Targets were specified by color with either the same or different colors for the right and left targets. The results indicated that asymmetric bimanual movements with different amplitudes (Experiment 1) or directions (Experiment 2) to targets of the same color were initiated more quickly than symmetric movements to different colors. The researchers concluded that the results indicate that interference occurred during target selection rather than at the motor programming stage. Two additional experiments investigated the interference associated with target selection by presenting distracting information. The results further supported that interference during the performance of bimanual action was related to target selection. These results were again confirmed and clarified in terms of the neural regions related to goal selection and movementrelated conflict in an experiment using fMRI (Diedrichsen, Grafton, Albert, Hazeltine, \& Ivry, 2006). The authors conclude that "bimanual movements may be difficult because of a cognitive limitation in selecting what to do with each hand and a limitation of the motor system in planning how to do so."

Over 30 years after the original Kelso et al. paper (1979a,b), Shea, Boyle \& Kovacs (2012) attempted to replicate the movement time data (control condition) from Kelso et al. (1979a,b) and determine if integrated feedback (Lissajous condition) would change the pattern of results (Fig. 4a-d). Examples of the display for the control condition and the Lissajous displays for the various unimanual and bimanual conditions are provided in Fig. $4 \mathrm{a}$ and $\mathrm{b}$, respectively. They found a pattern of results for the control condition that was remarkably similar to that observed in the Kelso et al. (1979a,b) experiments (Fig. 4c). That is, participants responded slower to the more difficult task when the movements were made with a single limb and when the two limbs moved to the same difficulty targets. However, when movements were made with one limb to the low ID and the other to the high ID, MTs for both limbs were consistent with 


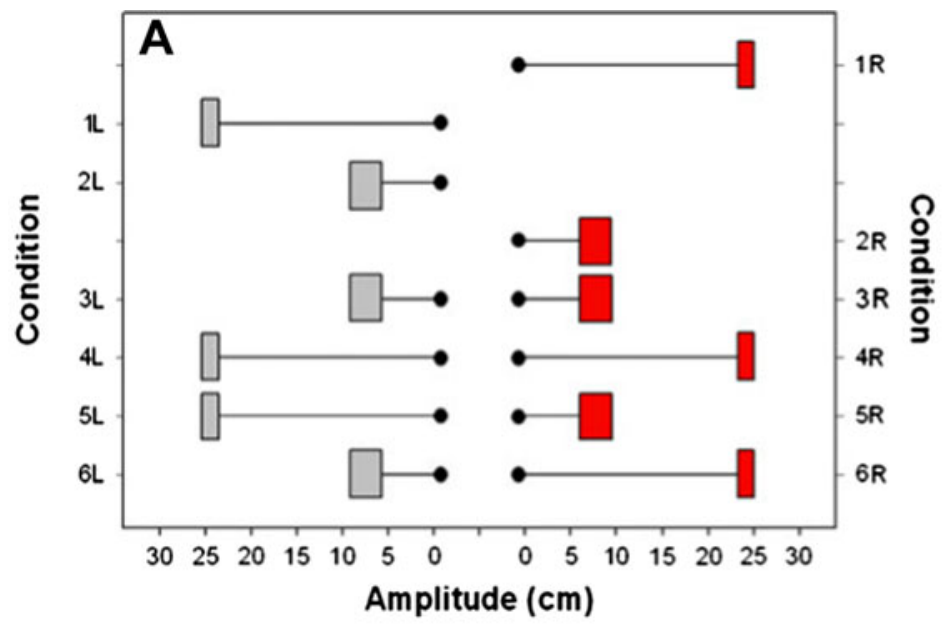

B
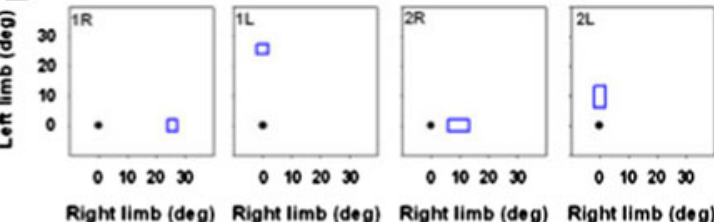

Right limb (deg) Right limb (deg) Right limb (deg) Right limb (deg)
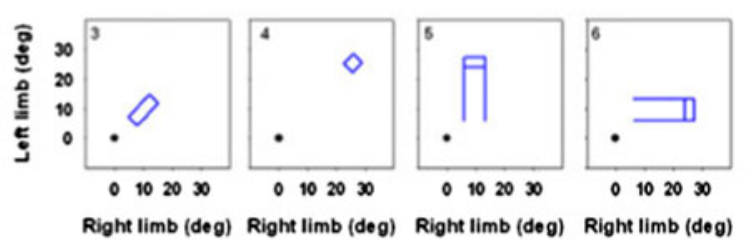

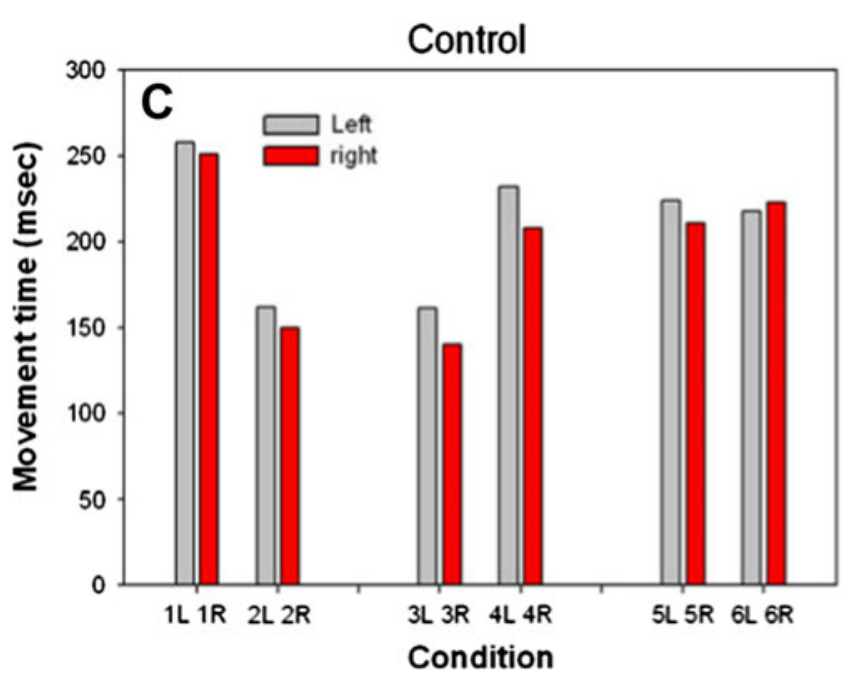

Fig. 4 Illustrations of the various ID conditions and mean movement time for the Control $(\mathbf{A}, \mathbf{C})$ and Lissajous $(\mathbf{B}, \mathbf{D})$ groups. Note that in the Lissajous condition movement times differed for the bimanual easy and

high ID requirements. Movement times in the Lissajous condition, when single limb movements were made and when bimanual movements were made to the same IDs, were similar to those observed in the control condition. However, participants that were provided Lissajous feedback and a template were able to simultaneously but differentially respond to low and high ID movements (Fig. 4d). Indeed, the movement times for the low and high difficulty movements in the mixed condition did not differ from the low and high difficulty movements with a single limb or the same ID movements in the bimanual conditions. These data suggest, as do the data from the more continuous bimanual tasks, that much of the difficulty associated with simultaneously producing discrete bimanual movements of different difficulties can be attributed to attentional and visual-perceptual factors typically available in the testing situation. Integration of the visual information in the form of a Lissajous plots and incorporation of the movement templates appear to allow participants to exploit the vast capabilities of the perception-action system.

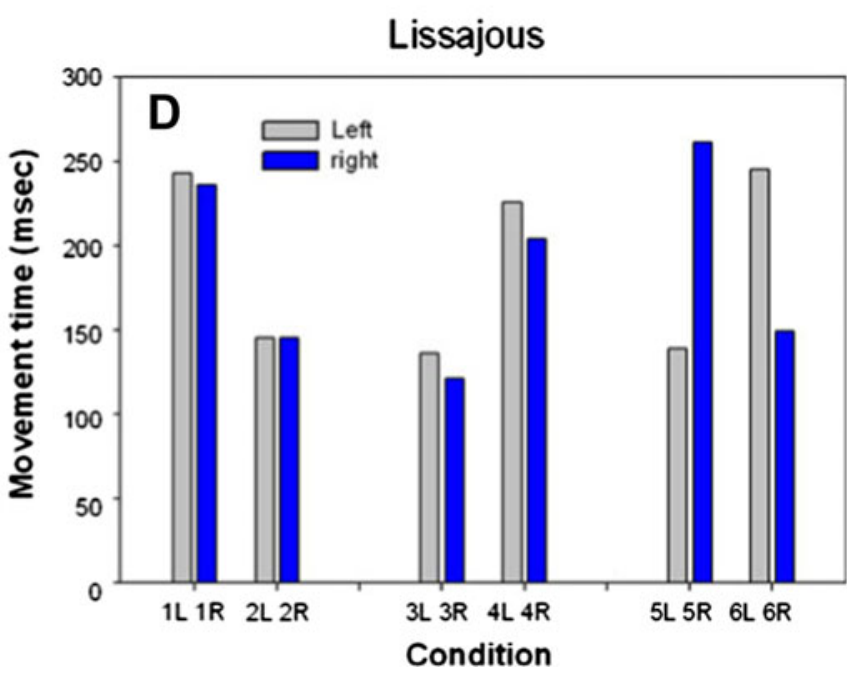

difficult tasks (Conditions $5 \mathrm{~L}$ and 5R; $6 \mathrm{~L}$ and 6R) but movement times were not different under the Control conditions (from Shea et al., 2012)

Wang, Kennedy, Boyle, and Shea (2013) recently combined discrete and reciprocal bimanual Fitts tasks of different difficulties to create what they considered an extreme challenge to the perceptual motor system. More specifically, the task required the participants to produce reciprocal movements $\left(32^{\circ}\right.$ amplitude, $2^{\circ}$ target width, ID $=5$ ) with the right limb and discrete movements $\left(16^{\circ}\right.$ amplitude, $4^{\circ}$ target width, ID $\left.=3\right)$ with the left limb. Although different ID requirements and different amplitudes were imposed on the two limbs, participants were able to effectively perform the complex bimanual coordination pattern with only $10 \mathrm{~min}$ of practice(Fig. 5a-c). Neither the movement times nor the percentage times to peak velocity for the low and high ID movements in the bimanual condition differed from those obtained from unimanual control conditions. These results using discrete and reciprocal tasks were consistent with what had been observed in Shea et al. (2012). Both experiments demonstrated that with 

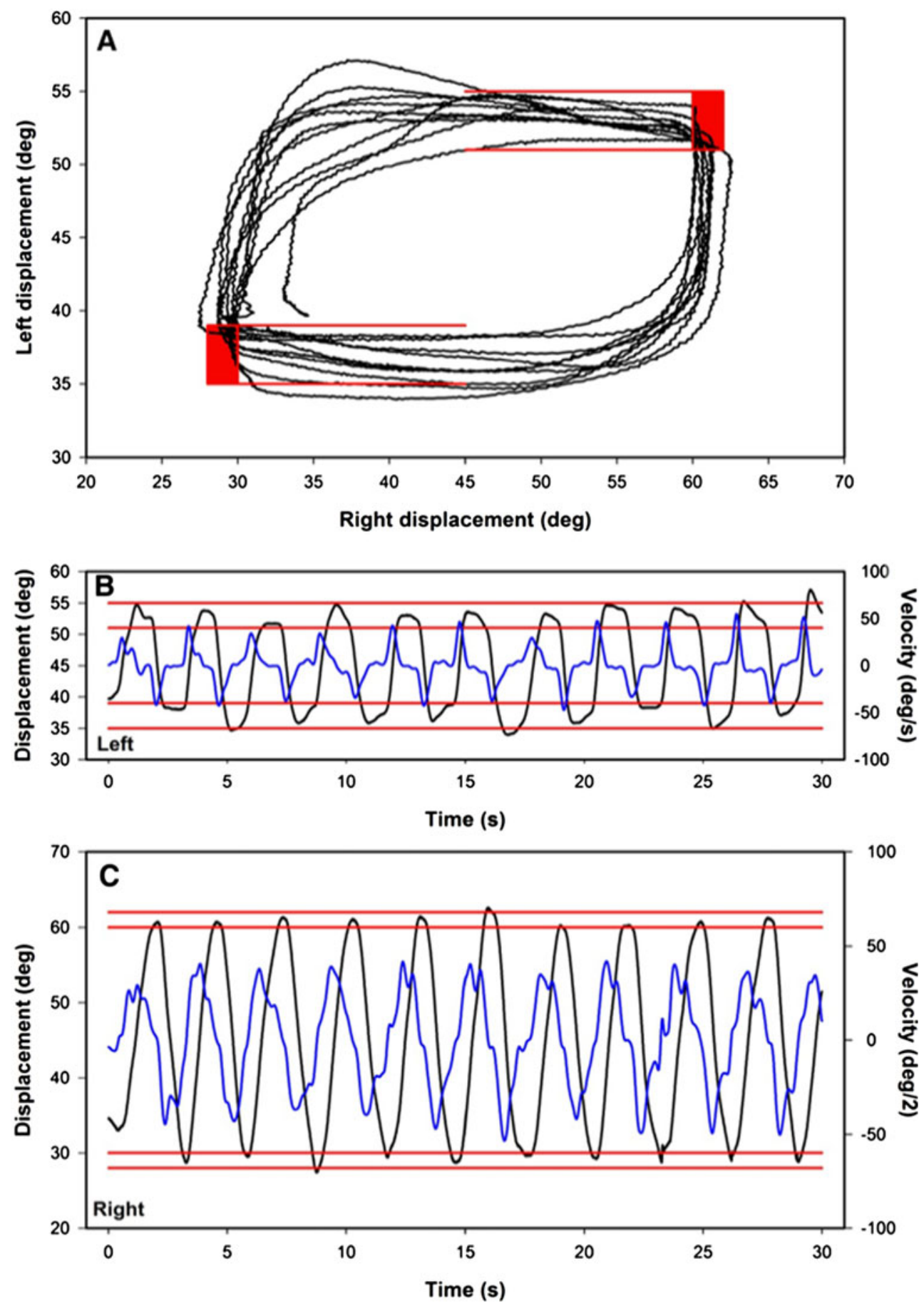

Fig. 5 Example of the time series for a typical participant in the Lissajous condition producing the continuous low ID movement with one limb and the discontinuous high ID movement with the contralateral limb. Lissajous plot (A), left (B) and right (C) limb displacements (black) and

Lissajous feedback the limb assigned the low ID moves faster than the limb assigned the high ID and the temporal characteristics of the velocity profiles are similar between the bi- and unimanual conditions for the respective limb. The same level of accuracy, as indicated by the endpoint variability and hits rates results, velocities (blue) for the test trial are provided. Note that the target areas are plotted on the respective limb displacement plot (from Wang et al., 2013)

between the bimanual and unimanual conditions also confirm that both limbs achieved the individual ID goals. The disparate amplitude goals achieved in this experiment were remarkable, especially when compared to the phenomenon of amplitude assimilation reported in previous bimanual coordination studies without the 
provision of integrated feedback information (Kelso et al., 1979a,b; Ryu \& Buchanan, 2004; Sherwood, 1994).

\section{Circle motion bimanual coordination}

While Lissajous plots with goal templates have been used with great success in experiments using reciprocal movements of the limbs, this type of feedback may not always provide the most salient information when circling movements are used. When Lissajous plots are used with circling movements only one dimension of the circle movement can be plotted for each limb. For example, the $y$-axis movement of the left limb and $x$ axis movement of the right limb could be plotted in the Lissajous plot with movements in the $x$-axis for the left limb and $y$-axis for the right limb disregarded. This could pose problems for the performer because the way in which the performer would correct an error would be different depending on where they are in the cycle. One solution to this problem is relatively simple when the radius of circle movement is fixed. That is, in circle movements with a fixed radius, it is relatively easy to determine relative angle and/or relative velocity on-line and use this information as direct feedback to the participant in much the same way as Lissajous feedback has been used. However, online relative angle or relative phase velocity would be difficult to determine in circledrawing experiments where participants are free to change the radius of the circles they draw (see Wilson, Snapp-Childs, Coats, \& Bingham, 2010 for a discussion of feedback issues).

This technique was used by Preilowski in 1972. In his experiment participants turned one crank with their left-hand counter clockwise and another with their right-hand counter clockwise (Fig. 6a). Movement of the cranks resulted in a line on an X-Y recorder to move from the bottom right up with the cranking with the left limb and left with the cranking of the right limb. Using this system, participants were quite effective in not only producing a 1:1 ratio but also $2: 1$ and 1:2. A similar strategy was used by Mechsner et al. (2001). They used two cranks which were geared differently and mounted under a table. In their experiment the movement of the cranks moved two flags mounted on the table top (see Fig. 6b). Because the cranks were geared differently when the flags moved in symmetry (1:1), the performer was actually producing a $4: 3$ coordination ratio. With this setup participants were able to produce this difficult bimanual coordination pattern relatively well with limited practice.

Boyle, Panzer, and Shea (2012) conducted a bimanual multi-frequency coordination experiment using circle movements in an attempt to demonstrate that a variety of multifrequency coordination patterns could be effectively performed when participants are provided on-line relative velocity information and a relative velocity goal. In essence this could be described as a more electronic version of the system used by Preilowski 40 years earlier. In this experiment, participants were assigned to one of five goal multi-frequency ratios $(1: 2,2: 3,3: 4,4: 5$, and 5:6), which were thought to represent increased difficulty. Participants were asked to produce the required multi-frequency ratio by continuously turning one manipulandum (counter-clockwise circular motion) with their left limb and the other (clockwise circular motion) with their right limb. The only difference between the ratio conditions was the goal relative velocity provided in the visual display. The goal was depicted as a straight line with a slope representing the specific relative velocity ratio to which the participant was assigned, with the difference in the left-hand velocity (slower moving) and right-hand velocity (faster moving) determining the slope. Boyle et al. hypothesize that participants provided this form of feedback would be able to effectively produce the various multi-frequency coordination patterns with little practice. Only 4 min of practice was provided for each task. The results indicated that participants very effectively performed each of the multi-frequency ratios with all three measures of overall performance (relative velocity, relative cycle frequency, and slope of the right-left limb
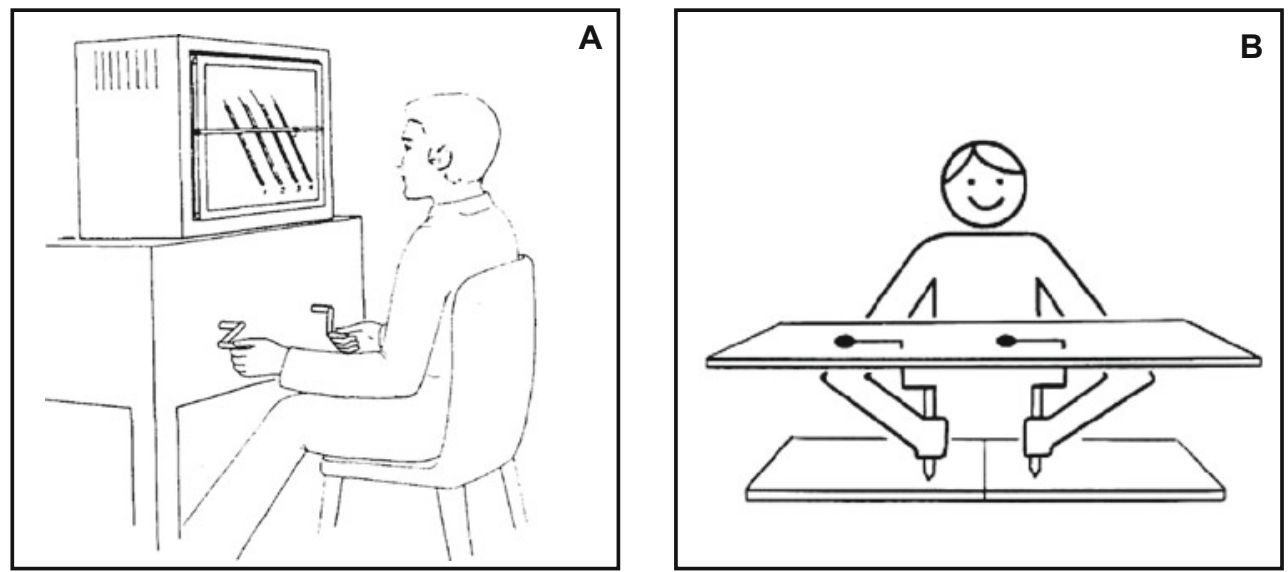

Fig. 6 Setup used by Preilowski (1972) to test multifrequency bimanual coordination with two normal subjects and two participants who had the corpus collosum sectioned 3 years earlier (A) and the set-up used by Mechsner et al. (2001) to test a 4:3 ratio (B) 
relative angle relationship), indicating very effective performance (Fig. 7a-e). In terms of unimanual measures, the results indicated that cycle durations for the right (faster moving) limb were shorter than for the left (slower moving) limb across all multi-frequency coordination tasks with the difference in cycle durations between the limbs decreasing as the goal ratio became more complex (e.g., 4:5, 5:6). In all cases, the cycle duration of the faster moving limb was maintained near the
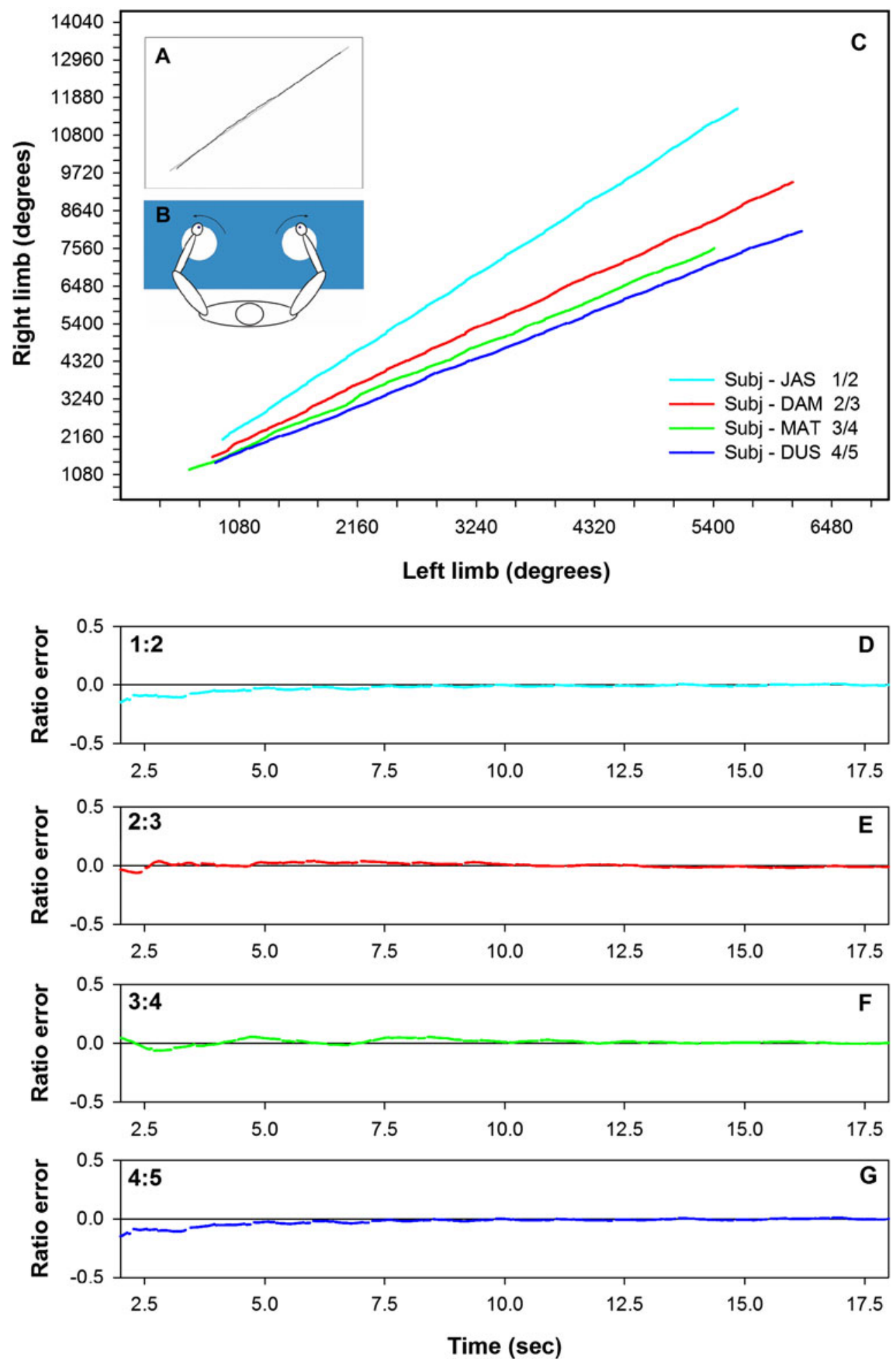

Fig. 7 The inset illustrates the feedback display (A) and participant performing circle movements with the manipulandum (B). Panel C illustrates examples of the various coordination patterns and the lower panels (B-E) illustrate examples of the coordination error time series for the respective condition illustrated in Panel C (from Boyle et al., 2012) 
$1 \mathrm{~Hz}$ target frequency. It must be noted that circling tasks are somewhat different from many bimanual tasks that just require motion about a single joint. Circling tasks often require upper-arm, forearm, and wrist motions. When tracing circles of different amplitudes, a tendency for the spontaneous emergence of multi-frequency patterns has been demonstrated (Buchanan \& Ryu, 2006; Buchanan \& Wang, 2012). These patterns, while often short-lived, can be stable for several epochs. However, when salient integrated feedback is provided participants can quickly detect and correct the ongoing coordination error.

\section{Tapping bimanual coordination patterns: Visual and auditory models}

The majority of research that has examined the influence of visual models on complex bimanual movements has focused on visual demonstration (Bingham, Schmidt, \& Zaal, 1999; Breslin, Hodges, Williams, Curran, \& Kremer, 2005; Hodges, Chua, \& Frank, 2003) and observational learning (Buchanan \& Dean, 2010, 2014; Maslovat, Hodges, Krigolson, \& Handy, 2010). More recently research has begun to focus on characteristics of the visual display itself (see Sigrist, Rauter, Riener \& Wolf, 2013 for review). Hu and Newell (2011), for example, manipulated the visual display in an attempt to determine how the amount of visual information regulates the coordination pattern in a bimanual asymmetric force production task. The amount of visual information was manipulated by changing the visual gain of the total force output displayed on a computer monitor.

Research has also examined the influence of auditory models on the production of complex motor tasks (see Sigrist et al., 2013 for review). The most well-known practical example is the Suzuki (1969) method of learning to play the violin. Individuals are taught to reproduce a musical score after they have been repeatedly exposed to a recording of the music. The recording of the music serves as an auditory model, and it is believed that the repeated exposure allows students to develop an internal representation that then can be used as a reference of correctness to guide their performance when they attempt to reproduce the musical score. Shea, Wulf, Park, and Gaunt (2001) examined the effects of an auditory model on the learning of relative and absolute timing of a unimanual tapping task. The auditory model consisted of a series of six beeps that corresponded to the temporal characteristics of the goal movement pattern. The results demonstrated that the auditory template had beneficial effects on the learning of relative timing structures. This result was consistent in follow-up studies using the same auditory model in different practice conditions (Han \& Shea, 2008; Lai, Shea, Bruechert, \& Little, 2002). Although previous research examining auditory models has yielded positive results (Han \& Shea, 2008; Lai et al., 2002; Shea et al., 2001), these results have not been systematically compared to visual and integrated models as an informational source prior to the performance of a complex tapping pattern. Additionally, research that has examined integrated visual and auditory models within the context of bimanual coordination and motor learning is limited. However, experiments have examined the effects of multisensory (auditory, visual, and tactile) cues during a unimanual tapping task (Elliott, Wing, \& Welchman, 2010) and simple and choice RT tasks (e.g., Girard, Collignon, \& Lepore, 2011; also see Miller, 1982). The results indicated that multisensory information was beneficial for sensorimotor synchronization and enhanced RT. It is important to note, however, that these studies did not include retention tests; therefore, it is not clear to what degree combined sensory information enhances learning.

An experiment by Kennedy, Boyle, and Shea (2013a,b) examined whether or not integrated salient task information (visual, auditory, or auditory-visual models) presented prior to each acquisition trial would enhance the development of an internal representation of a goal rhythm that could be used to maintain performance when the model was withdrawn. On each trial during acquisition, the computer generated a visual display and/or played a pattern of notes that described the goal coordination pattern. This information was removed prior to the signal indicating that the participants should attempt to produce the goal movement pattern. However, during the trial a participant's tapping pattern would generate visual and/or auditory stimuli, depending on the model condition, with the temporal characteristics determined by their actual performance and not the goal pattern (Fig. 8). For example, in the auditory model condition during acquisition, each time they tapped with their left hand a tone would be played and each time they tapped with their right hand a different tone was played. These tones had the same sound characteristics as those used in the auditory model played prior to each acquisition trial. In this way, the goal pattern was never presented during the trial, but the participant was encouraged to use that prior information to help guide their bimanual tapping pattern. The authors proposed that this method of providing information would not only enhance acquisition performance, but also free the participant from any dependency on the model information. However, the authors were not sure whether auditory, visual, or combined auditory-visual model information would provide the participant the most salient information in learning the tapping task. Modeling studies have demonstrated that visual models tend to help build relative relationships between movement events (e.g., Buchanan et al., 2008; Buchanan \& Wright, 2011; Buchanan \& Dean, 2014; Lai et al., 2002; Scully \& Newell, 1985) while auditory models tend to enhance absolute temporal characteristics of the movements (e.g., Shea et al., 2001). If these differential effects were true, they predicted that the combined auditory-visual model conditions would lead to optimal performance and learning. Note that no direct online error information was provided, but 

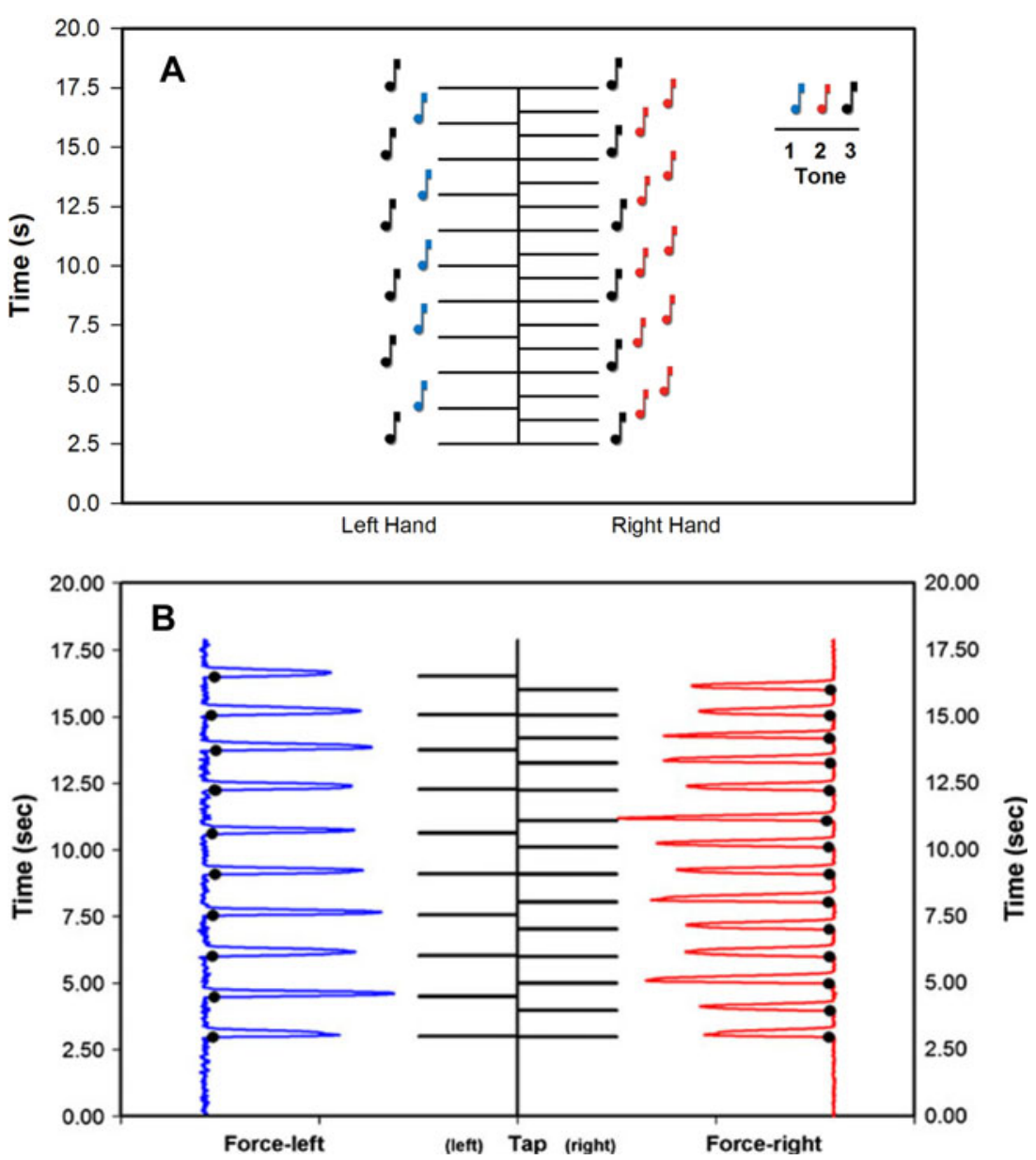

Fig. 8 Example of the auditory-visual model (A) used in Kennedy et al. (2013a). Note in this model the lines and tones appeared in temporal order from the bottom to the top during the pretrial interval. An example is given of the left and right limb forces produced by tapping on the respective load cell for one participant in the visual/auditory model condition (B). The blue line (left) represent the force time series for the left hand, and the red (right) is the force time series for the right hand. Note that the forces were not presented in the display provided to the participants. The dots on the force time series indicate the point at which a tap was detected.

participants could use an internal auditory or visual reference developed from the model to determine when errors occur and to aid in the planning of strategies to correct and/or minimize subsequent errors.

The accuracy with which the goal ratio was achieved for visual, auditory, and visual/auditory model conditions was substantially increased across practice for all groups. That is, participants minimized their errors in producing the goal ratio by the end of practice relative to the beginning of practice. This was coupled with the substantial reductions in the intertap variability of both the left and the right hands for all model groups but not for the metronome control group. Indeed, performance of the model groups at the end of practice was similar to that on the retention test. This exemplifies the powerful effect of the various model conditions in quickly directing the participants to produce three taps with the right hand for every
The horizontal lines to the left of center represent the time at which lefthand force onset was detected, and horizontal lines to the right of center represent the time at which right-hand force onset was detected. These lines were presented online to the participant during the course of the trial as each tap was made. Simultaneous with the appearance of the line, the tone associated with that limb tap was played. Thus, the participant would try to rebuild the display and replay the tones presented during the pretrial period

two taps of the left hand while maintaining on average approximately the time intervals represented in the visual model or indicated by the auditory model. Performance differences were noted as a result of practice in the measures of ratio accuracy and inter-tap variability. Remarkably, the decreases in ratio errors and inter-tap interval variability were generally carried over to the retention test where model was withdrawn. However, the withdrawal of the metronome resulted in increases in ratio error for the metronome group. This is consistent with the notion that learning was enhanced under the model conditions relative to the metronome condition.

These results provide further evidence that perceptual and attentional constraints imposed by the testing environment play a role in the difficulty associated with the performance of complex polyrhythms. When practice conditions are imposed that reduce these constraints, performers are able to 
quickly and effectively perform the desired coordination pattern. In the tapping experiment by Kennedy et al. (2013a) this was done by using visual and auditory models.

\section{Emerging picture}

The perceptual motor system is capable of dissociating the interaction (inherent coupling) between the two limbs to effectively produce a wide range of bimanual coordination patterns. That is, the two limbs can produce distinctly different discrete and reciprocal movement patterns in order to produce a single coordinated bimanual pattern. These results agree with the notion of Mechsner et al. (2001) and Franz, Zelaznik, Swinnen, and Walter (2001) that perceptual influences can sometimes override action constraints in coordinated bimanual movements. When provided appropriate perceptual feedback, people can overcome or otherwise bypass a variety of bimanual coordination constraints. For example, neural crosstalk (e.g., Kennerley et al., 2002; Swinnen, 2002) and forward-command stream (e.g., Carson \& Kelso, 2004) are thought to restrict an individual's ability to produce complex discrete-reciprocal bimanual coordination movements without the aid of integrated feedback and an optimal movement path provided to them in the display. When this type of display is provided participants can easily detect and correct coordination errors and effectively produce the desired coordination pattern with relatively little practice. Debaere et al. $(2001,2003)$ provides evidence that participants utilize different neural pathways when this form of integrated feedback is provided with this pathway less restrictive to bimanual movements than when participants attempt to generate these coordination patterns without the aid of feedback.

As noted in the introduction, the use of integrated displays seems to promote what has been termed an external focus of attention. A rapidly growing literature on attentional focus (see Wulf, 2007, 2013, for recent reviews) has demonstrated that when participants focus on the movements of their limbs (internal focus) while learning or performing a motor skill perform and learn more poorly than participants who focus on the outcome (external focus) of their movement. This has been demonstrated for a large variety of tasks (e.g., McNevin et al., 2003; Wulf, Lauterbach, \& Toole, 1999; Wulf \& Su, 2007). The benefit of external versus internal focus of attention is thought to result because participants instructed to focus on the movement of their limbs attempt to actively intervene in the ongoing control processes required to produce the movement. In this regard, McNevin et al. (2003) proposed the constrained action hypothesis. This hypothesis was based on two main findings: First, when participants attempted to maintain balance on a stabilometer under internal focus of attention instructions, the frequency characteristics (mean power frequency, MPF) of the platform's motion decreased and the average deviation from horizontal (RMSE) increased. Second, when instructions that focused attention externally were provided, the frequency characteristics of the platform's motion increased and deviations from horizontal decreased. The slowing down of the corrective actions under internal focus was thought to result from active intervention of the participants in the control processes with this type of control inhibiting more natural, reflective control processes typically responsible for balance control. In the present experiment, attentional focus instructions were not provided to the participants. However, participants provided vision of their limbs may have directed some of their attentional resources to their limbs and away from the Lissajous plots. This could result in a shift from an external to internal focus of attention. Participants not permitted vision of their limbs would certainly be less likely to adopt an internal focus of attention. Indeed, a number of participants indicated at the end of the experiment that they were not aware that horizontal movement of the cursor resulted from movement of the right limb and vertical movement of the cursor resulted from left-hand movement. In addition, some participants not provided vision of their limbs indicated that when they attempted to monitor what their arms were doing, performance was negatively impacted so they tried to keep their attention direct to the display.

\section{Positive effects of integrated feedback}

The recent findings using integrated Lissajous, relative velocity, and auditory/visual model displays are consistent with other recent bimanual coordination research (Mechsner et al., 2001), visuo-motor tracking research (e.g., Wilson et al., 2005a,b; Ryu \& Buchanan, 2009), and even rapid aiming research constrained by Fitts' Law (e.g., Kovacs, Buchanan, \& Shea, 2008; Wang et al., 2013) which report that salient perceptual information can override some aspects of the system's intrinsic dynamics typically linked to motor output control. These findings suggest that the strong tendencies toward mirror symmetry and homologous muscle activation found in numerous previous bimanual movement studies and the difficulties in producing harmonic ratios and polyrhythms may actually represent detrimental effects attributable to the perceptual information available in the environment and the attentional demands resident in attempting to monitor multiple sources of information and/or attempting to do two different things at the same time. Given reduced attention demands and integrated, salient perceptual information participants can effectively produce complex bimanual coordination patterns with little or no practice. Thus, even as the intrinsically stable in-phase and anti-phase patterns constrain the production of certain 1:1 coordination patterns and multi-frequency patterns in the absence of salient augmented feedback, the appropriate use of integrated feedback representations, where attentional demands are minimized, removes a large portion of the impact 
of the constraints and allows the performer to effectively produce with little practice typically unstable coordination patterns (Fig. 9a,b).

The important feature of the displays provided in recent experiments was that the displays facilitated error detection and correction processes. This allowed for a very rapid and effective reduction in coordination errors in a wide variety of bimanual coordination patterns. We do not believe that these displays eliminate or otherwise block intrinsic constraints such as cortical and subcortical neural crosstalk, coupling, or phase attraction that tend to pull the system towards in-phase and anti-phase coordination. Rather we are of the opinion the various displays used in the experiments clearly decreased the negative influence of these factors. The display seemed to provide a means whereby the link connecting visual and proprioceptive feedback to the organization of outgoing forward based motor commands could be established such that the interplay between perceptual and action constraints were facilitated by the integrative action of the nervous system and not overly constrained by any one factor. In very simple terms, when the available perceptual information allows the performer to determine when they are making an error and provides direction on how to correct this error, performance can be rapidly and effectively achieved even if the system is regularly perturbed by neural crosstalk, for example. The rapidity with which the current perceptually defined patterns were mapped onto the perception-action system indicates that the system's capabilities are extensive. Taken together, these results emphasize that a true understanding of the "design of the brain" can only be forthcoming when the roles of both the action components (e.g., Kelso \& de Guzman, 1988) and the perceptual components (e.g., Mechsner et al., 2001) are integrated in a true perception-action dynamics perspective (see Atchy-Dalama, Peper, Zanone, \& Beek, 2005; Bingham 2004a,b; Bingham et al., 1999; Carson \& Kelso, 2004; Swinnen \& Wenderoth, 2004; Zaal, Bingham, \& Schmidt, 2000). The findings show that stable perception-action patterns can emerge when the available environmental information provides guidance to the participant as to the goal pattern and can be used to assist the detection and correction of coordination errors when they occur. Early work demonstrated that the scaling of non-specific environmental information can induce phase transitions from unstable to stable coordination patterns (Kelso, 1984; Buchanan \& Kelso, 1993). It was then shown that specific environmental information can through extensive practice lead to the formation of new stable patterns that can be produced when the training information is removed. The studies reviewed in the present manuscript as a group show that environmental information when presented in specific ways can facilitate the effective production of difficult and complex bimanual patterns. However, the use of the various integrated displays does come with certain restriction as discussed below.

\section{Reducing potential negative consequences of integrated concurrent feedback}

A critical feature of many of the experiments highlighted in this manuscript is that in some cases participants can become highly dependent on the information provided by concurrent visual displays. Indeed, as noted earlier, Kovacs et al. (2009a) found that continuous relative phase error and variability were
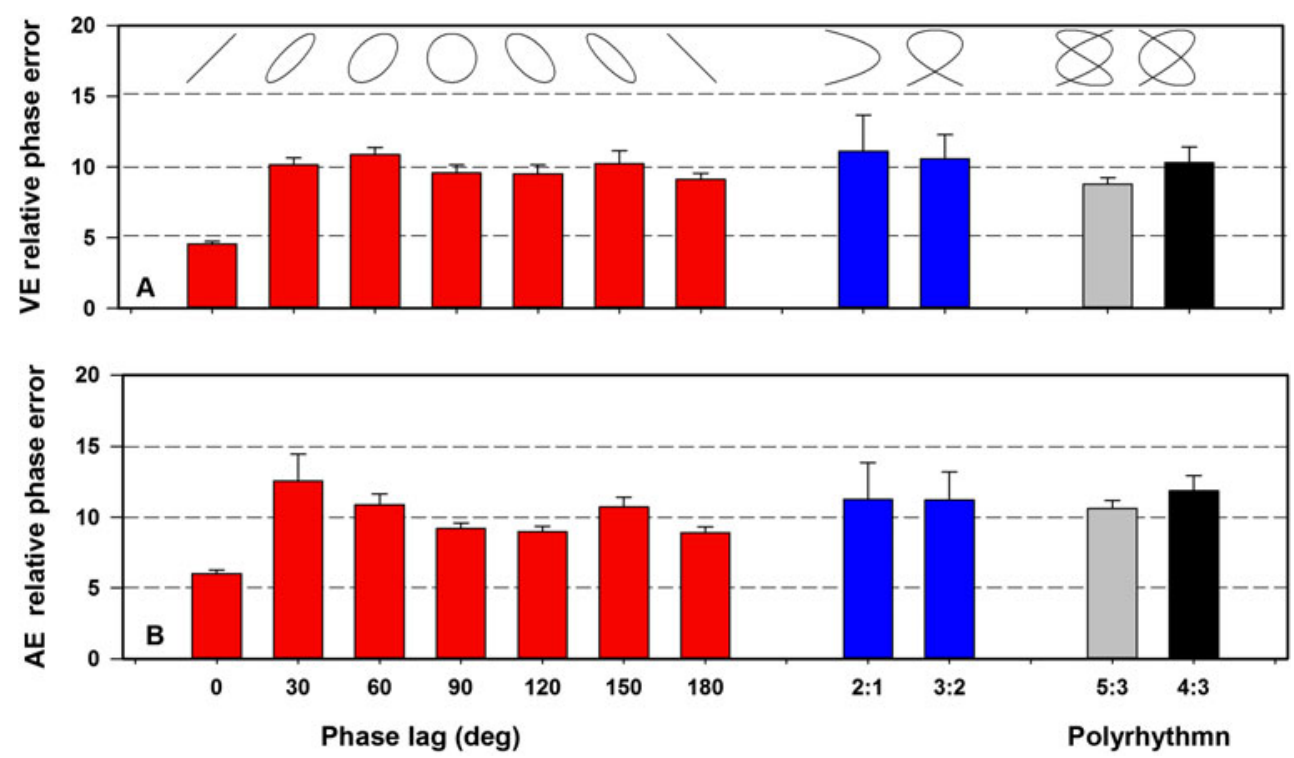

Fig. 9 Variability (A) and absolute error (B) in continuous relative phase from the Kovacs et al. (2010b) experiment are compared with experiments using similar protocols testing phase lags of $0-180$ in 30 increments (Kovacs et al. 2009a) and multi-frequency (2:1 and 3:2, Kovacs

et al. 2010a) bimanual coordination patterns. Note that the shapes of the Lissajous templates used for the various conditions are provided above the variability bars in A (from Kovacs et al., 2010b) 
remarkably low when tested after only 5 min of practice when concurrent Lissajous feedback was available, but not when the Lissajous feedback was withdrawn. This finding suggests that the Lissajous plot with cursor and template provided participants a means by which they were able to detect their coordination errors and perform the necessary corrections. This would be considered a short-term adaptation using online feedback control because on a subsequent test when the Lissajous feedback was removed error and variability in relative phase increased significantly. This increase indicated that participants had not developed an internal representation or changed the underlying attractor landscape, but had learned to use the concurrent information provided to effectively perform the required coordination pattern. In other words, participants were dependent on the concurrent feedback indicating their movements were externally, not internally, driven. The notion of externally and internally driven bimanual response production is not new. Debaere et al. (2001, 2003) have provided evidence that suggests the existence of distinct cortico-cortical and sub-corticocortical neural pathways for externally (augmented feedback) and internally guided cyclical bimanual movements. Presumably, the externally guided movements are dependent on the feedback available in the testing environment, while internally guided movements are not dependent on this type of extrinsic feedback.

In the motor learning literature information provided by extrinsic feedback regarding the progress and outcome of movements acquired through concurrent and terminal feedback is perceived as one of the most important variables in the learning of motor skills (Adams, 1971; Bilodeau \& Bilodeau, 1958). Indeed, the feedback literature is replete with examples of benefits of providing salient information to the learner. However, this literature also chronicles the strong tendency for participants to develop dependencies on various types of concurrent and terminal feedback. In an attempt to characterize the research on feedback and to offer potential strategies to minimize problems associated with feedback withdrawal, Salmoni, Schmidt, and Walter (1984) proposed the guidance hypothesis. The guidance hypothesis postulates that although frequent feedback provided during practice is very powerful in guiding the learner toward the correct response, it also results in a dependency on that feedback and blocks the processing of other important sources of information (Bjork, 1988; Schmidt, 1991; Schmidt \& Wulf, 1997) that are necessary to develop an internal representation of the movement task that is capable of producing the movement when feedback is withdrawn. One of the methods used to reduce participants' dependencies on extrinsic feedback is to reduce the percentage of trials in which the extrinsic information is provided (e.g., Lee, White, \& Carnahan, 1990; Sparrow \& Summers, 1992; Weeks, Zelaznik, \& Beyak, 1993; Winstein \& Schmidt, 1990). One method of reducing feedback that has shown promise involves decreasing the amount feedback provided as practice progresses. This tactic has been termed a fading schedule.

Kovacs and Shea (2011) designed an experiment using a $1: 1$ with $90^{\circ}$ phase shift bimanual coordination task to determine if systematically reducing the percentage of time that Lissajous display was provided during practice results in test performance without concurrent feedback being maintained at levels observed when Lissajous displays were continually provided. In this experiment participants attempted to coordinate the movements of their arms in an attempt to produce the required phase relationship while being provided concurrent Lissajous displays with $100 \%, 50 \%$, or $0 \%$ frequency. Participants were provided 5,10 , or $20 \mathrm{~min}$ of practice in their respective feedback conditions. As previous research has shown (Kovacs et al., 2009a,b), after only 5 min of practice participants receiving $100 \%$ Lissajous feedback were very effective (low relative phase error and variability) in producing the required coordination pattern. However, when the feedback was withdrawn error and variability of relative phase substantially increased, indicating an absence of an effective internal representation of the coordination pattern. The same pattern of results was observed for the $100 \%$ Lissajous groups after 10 and $20 \mathrm{~min}$ of practice. Thus, regardless of the amount of practice, participants in the $100 \%$ feedback condition did not improve on a delayed test when Lissajous displays were not available. This result in itself is quite surprising considering the ubiquitous finding that more practice benefits learning. It is possible that the availability of the Lissajous display on each trial prompted participants to rely on this source of information to guide their movements and the processing of the information derived from the Lissajous display essentially blocked the participants from processing other more intrinsic information necessary for acquiring an internal representation of the task (e.g., Blandin, Toussaint, \& Shea, 2008; Schmidt \& Bjork, 1992). Indeed, the guidance hypothesis (Salmoni et al., 1984) postulates that on practice trials where extrinsic feedback is withheld in reduced frequency of feedback conditions (i.e., $50 \%$ ) participants seek out and process other sources of information necessary to perform the task. This additional processing is thought to result in participants acquiring the capability to detect and correct one's own errors independent of the extrinsic feedback available. Indeed, in the Kovac and Shea (2011) study participants in the $50 \%$ condition performed remarkably well when the Lissajous display was available during acquisition and when the Lissajous display was withdrawn on the retention test. This suggests that during practice when the Lissajous display was withdrawn participants sought out other sources of information and internalized the information provided while the display was available. The result was effective performance with and without the Lissajous display. Note that using this technique of systematically reducing the display information that still only a few minutes of practice was required to effectively perform and 
learn the goal 1:1 with $90^{\circ}$ phase offset. This is quite remarkable when considering that previous experiments (e.g., Buchanan, 2004; Lee, Swinnen, \& Verschueren, 1995; Zanone \& Kelso, 1992) provided significantly more practice to achieve similar levels of performance.

In an experiment by Buchanan and Wang (2012), the researchers simply displayed the cursor in one Lissajous display and the Lissajous template on another display. This tactic was designed to encourage the internalization of the environmental information, provide the participant with the necessary information to detect and correct errors, and ultimately reduce the participants' reliance on the extrinsic displays. Following only 5 min of practice, effective performance of a 2:1 bimanual coordination pattern without the augmented Lissajous plot was achieved. Thus, rapid encoding of a complex motor task can occur if the use of attentional resources is optimized. In the Buchanan and Wang (2012) study, it was argued that the shifting of the cursor from the Lissajous template required more attentional effort to be directed towards other intrinsic sources of information, in turn facilitating the development of a motor representation capable of sustaining performance when the Lissajous feedback was withdrawn. It should also be noted that the visual, auditory, and visual/auditory model techniques used by Kennedy et al. (2013a) during acquisition were removed on the retention test. The result was that performance on the retention test with the model information was quite effective. It is also important to note that in the tapping experiment by Kennedy et al. (2013a) where visual, auditory, and visual/auditory models were used, the model was presented prior to each trial but was not available during the production of the tapping task. This prompted the participant to build an internal representation of the task that could be used to detect and correct errors during the production of the task. The bottom line is that integrated displays (e.g., Lissajous) with goal templates can be used to greatly accelerate the learning of a wide variety of bimanual coordination patterns. An important feature of this type of experiment in the future will be to design new practice protocols and displays that accelerate performance improvement while at the same time enhance the development of an internal representation that will allow performance to be maintained with and without the extrinsic sources of information.

There remain, however, important theoretical challenges for future researchers in bimanual coordination. In light of recent findings, future researchers on bimanual coordination should determine if the dynamical systems perspective, traditionally used to describe and explain the coupling and synergistic characteristics of bimanual coordination, continues to be the most effective theoretical perspective to drive our understanding of these tasks and the interplay between perceptual and action constraints, or would it in the long run be more productive to move toward broader theoretical perspectives (e.g., cognitive neuroscience perspective by Ivry and colleagues (Ivry et al., 2004), or central executive of Baddeley (e.g., 1992, 1996).
One of many questions that needs to be answered in determining which theoretical perspective would be most appropriate is whether the perceptual and action constraints can be viewed in a hierarchical manner or are better described in terms of dynamic self-organizing systems where the organization emerges as a result of the external conditions and internal dynamics. Recent data suggest a type of cognitive/perceptual dominance over lower level action constraints whereby perceptual constraints can be minimized via various display techniques in such a way as to counteract the inherent intrusions of the remaining action constraints. The degree to which perception and action constraints are shown to be dissociable will play a role in this conversation.

\section{Summary}

The production of a large variety of bimanual coordination patterns are plagued with a coalition of perceptual and action constraints that limit a performer's ability to coordinate different movements of the two limbs, and have led researchers to conclude that the "limbs are constrained to act as a single unit" (Kelso et al., 1979a,b). While substantial practice can allow performers to overcome some of these constraints for relatively simple bimanual coordination patterns, other more difficult patterns have appeared to be almost impossible to effectively perform. However, the recognition of these constraints has led researchers to find methods to limit the impact of the perceptual constraints allowing a wide variety of bimanual coordination patterns, once thought to be nearly impossible to perform, to be effectively performed with only minimal practice. These methods, while effective, do not limit the action constraints related to cortical and subcortical neural crosstalk, but do provide the performer with integrated information necessary to minimize the impact of intrinsic action constraints. What appears to be necessary is to provide information to the performer that allows them to easily detect and correct coordination errors when they occur. Effective methods that allow the detection and correction of coordination errors are varied. These findings provide strong evidence that the same bimanual coordination requirements may be relatively easy to perform and learn in one context and nearly impossible in another context.

\section{References}

Adams, J. A. (1971). A closed-loop theory of motor learning. Journal of Motor Behavior, 3, 111-150.

Allen, M. (1983). Models of hemispheric specialization. Psychological Bulletin, 93, 73-104.

Amazeen, D. L., Amazeen, P. G., Treffner, P. J., \& Turvey, M. T. (1997). Attention and handedness in bimanual coordination dynamics. 
Journal of Experimental Psychology: Human Perception and Performance, 23, 1552-1560.

Amazeen, E. L., DaSilva, F., \& Amazeen, P. G. (2008). Visualspatial and anatomical constraints interact in a bimanual coordination task with transformed visual feedback. Experimental Brain Research, 191, 12-24.

Aschersleben, G., \& Prinz, W. (1995). Synchronizing actions with events: The role of sensory information. Perception and Psychophysics, 57(3), 305-317.

Atchy-Dalama, P., Peper, C. E., Zanone, P. G., \& Beek, P. J. (2005). Movement-related sensory feedback mediates the learning of a new bimanual relative phase pattern. Journal of Motor Behavior, 37, 186-196.

Baddeley, A. (1992). Working memory. Science, 255, 556-559.

Baddeley, A. (1996). Exploring the central executive. Quarterly Journal of Experimental Psychology: Human Experimental Psychology, 49, 5-28.

Baldissera, F., Cavallari, P., Marini, G., \& Tassone, G. (1991). Differential control of in-phase and anti-phase coupling of rhythmic movements of ipsilateral hand and foot. Experimental Brain Research, 83, 375-380.

Beek, P. J., Peper, C. E., \& Stegeman, D. F. (1995). Dynamical models of movement coordination. Human Movement Science, 14, 573-608.

Bilodeau, E. A., \& Bilodeau, I. M. (1958). Variable frequency of knowledge of results and the learning of a simple skill. Journal of Experimental Psychology, 55, 379-383.

Bingham, G. P. (2004a). A perceptually driven dynamical model of bimanual rhythmic movement (and phase perception). Ecological Psychology, 16, 45-53.

Bingham, G. P. (2004b). Another timing variable composted of state variables: Phase perception and phase driven oscillators. In $\mathrm{H}$. Hecht \& G. I. P. Savelsbergh (Eds.), Advances in Psychology: Time to Contact. Amsterdam: Elsevier.

Bingham, G. P., Schmidt, R. C., \& Zaal, F. T. J. M. (1999). Visual perception of relative phasing in human limb movements. Perception and Psychophysics, 61, 246-258.

Bjork, R. A. (1988). Retrieval practice and the maintenance of knowledge. In M. M. Gruenberg, P. E. Morris, \& R. N. Sykes (Eds.), Practical aspect of memory (IIth ed., pp. 396-401). London: Wiley.

Blandin, Y., Toussaint, L., \& Shea, C. H. (2008). Specificity of practice: Interaction between concurrent sensory information and terminal feedback. Journal of Experimental Psychology: Learning, Memory and Cognition, 34, 994-1000.

Bogacz, S. (2005). Understanding how speed affects performance of polyrhythms: Transferring control as speed increases. Journal of Motor Behavior, 37, 21-34.

Boonstra, T. W., Daffertshofer, A., Breakspear, M., \& Beek, P. J. (2007). Multivariate time-frequency analysis of electromagnetic brain activity during motor learning. Neuroimage, 36, 370-377.

Boyle, J., Panzer, S., \& Shea, C. H. (2012). Increasingly complex bimanual multi-frequency coordination patterns are equally easy to perform with on-line relative velocity feedback. Experimental Brain Research, 216, 515-525.

Breslin, G., Hodges, N. J., Williams, A. M., Curran, W., \& Kremer, J. (2005). Modeling relative motion to facilitate intra-limb coordination. Human Movement Science, 24, 446463.

Buchanan, J. J. (2004). Learning a single limb multi-joint coordination pattern: The impact of a mechanical constraint on the coordination dynamics of learning and transfer. Experimental Brain Research, 156, 39-54.

Buchanan, J. J., \& Kelso, J. A. S. (1993). Posturally induced transitions in rhythmic multijoint limb movements. Experimental Brain Research, 94, 131-142.
Buchanan, J. J., \& Dean, N. (2010). Specificity in practice benefits learning in novice models and variability in demonstration benefits observational practice. Psychological Research, 74, 313-320.

Buchanan, J. J., \& Dean, N. (2014). Consistently modeling the same movement strategy is more important than model skill level in observational learning contexts. Acta Psychologica, 146, 19-27.

Buchanan, J. J., \& Ryu, Y. U. (2005). The interaction of tactile information and movement amplitude in a multijoint bimanual circle-tracing task: Phase transition and loss of stability. Quarterly Journal of Experimental Psychology, 58, 769-787.

Buchanan, J. J., \& Ryu, Y. U. (2006). One to one and polyrhythmic temporal coordination in bimanual circle tracing. Journal of Motor Behavior, 38, 163-184.

Buchanan, J. J., \& Ryu, Y. U. (2012). Scaling movement amplitude: Adaptation of timing and amplitude control in a bimanual task. Journal of Motor Behavior, 44(3), 135-147.

Buchanan, J. J., \& Wang, C. (2012). Overcoming the guidance effect in motor skill learning: Feedback all the time can be beneficial. Experimental Brain Research, 219, 305-320.

Buchanan, J. J., \& Wright, D. L. (2011). Generalization of action knowledge following observational learning. Acta Psychologica, 136, $167-178$.

Buchanan, J. J., Ryu, Y. U., Zihlman, K., \& Wright, D. L. (2008). Observational practice of relative but not absolute motion features in a single limb mutli-joint coordination task. Experimental Brain Research, 191, 157-169.

Cardoso de Oliveira, S., Gribova, A., Dochin, O., Bergman, H., \& Vaada, E. (2001). Neural interaction between motor cortical hemispheres during bimanual and unimanual arm movements. European Journal of Neuroscience, 14, 1881-1896.

Carson, R. G. (1989). Manual asymmetries - In defense of a multifactorial account. Journal of Motor Behavior, 21, 157-162.

Carson, R. G. (2005). Neural pathways mediating bilateral interactions between the upper limbs. Brain Research Reviews, 49, 641-662.

Carson, R. G., Byblow, W. D., Abernethy, B., \& Summers, J. J. (1996). The contribution of inherent and incidental constraints to intentional switching between patterns of bimanual coordination. Human Movement Science, 15, 563-589.

Carson, R. G., \& Kelso, J. A. S. (2004). Governing coordination: Behavioural principles and neural correlates. Experimental Brain Research, 154(3), 267-274.

Cattaert, D., Semjen, A., \& Summers, J. J. (1999). Simulating a neural cross-talk model for between-hand interference during bimanual circle drawing. Biological Cybernetics, 81, 343-358.

Collier, G. L., \& Wright, C. E. (1995). Temporal rescaling of simple and complex ratios in rhythmic tapping. Journal of Experimental Psychology: Human Perception and Performance, 21, 602-627.

de Poel, H. J., Peper, C. E., \& Beek, P. J. (2008). Laterally focused attention modulates asymmetric coupling in rhythmic interlimb coordination. Psychological Research-Psychologische Forschung, 72(2), 123-137.

Debaere, F., Swinnen, S. P., Beatse, E., Sunaert, S., Van Hecke, P., \& Duysens, J. (2001). Brain areas involved in interlimb coordination: A distributed network. Neuroimage, 14, 947-958.

Debaere, F., Wenderoth, N., Sunaert, S., Van Hecke, P., \& Swinnen, S. P. (2003). Internal vs external generation of movements: Differential neural pathways involved in bimanual coordination performed in the presence or absence of augmented visual feedback. Neuroimage, 19, 764-776.

Deutsch, D. (1983). The generation of two isochronous sequences in parallel. Perception and Psychophysics, 34, 331-337.

Diedrichsen, J., Hazeltine, E., Kennerley, S., \& Ivry, R. B. (2001). Moving directly cued location aboloshes spatial interference bimanual actions. Psychological Science, 12, 493-498.

Diedrichsen, J., Ivry, R. B., Kennerley, S., Hazeltine, E., \& Cohen, A. (2003). Bimanual interference associated with the selection of target 
locations. Journal of Experimental Psychology: Human Perception and Performance, 29, 64-77.

Diedrichsen, J., Grafton, S., Albert, N., Hazeltine, E., \& Ivry, B. (2006). Goal-selection and movement-related conflict during bimanual reaching movements. Cerebral Cortext, 16, 1729-1738.

Elliott, M. T., Wing, A. M., \& Welchman, A. E. (2010). Multisensory cures improve sensorimotor synchronization. European Journal of Neuroscience, 31, 1828-1835.

Fowler, C. A., \& Turvey, M. T. (1978). Skill acquisition: An event approach with special reference to searching for the optimum of a function of several variables. In G. Stelmach (Ed.), Information processing in motor learning and control (pp. 2-40). New York: Academic Press.

Fraisse, P. (1946). Contribution a etude du rythme en tant que forme temporelle. Journal de Psychologie Normale et Pathologique, 39, 283-304.

Franz, E. A., Zelaznik, H. N., Swinnen, S. P., \& Walter, C. (2001). Spatial conceptual influences on the coordination of bimanual actions: When a dual task becomes a single task. Journal of Motor Behavior, 33, 103-112.

Fuchs, A., \& Jirsa, V. K. (Eds.). (2008). Coordination: Neural, Behavioral and Social Dynamics. Heidelberg: Springer.

Girard, S., Collignon, O., \& Lepore, F. (2011). Mutlisensory gain within and across hemispaces in simple and choice reaction time paradigms. Experimental Brain Research, 214, 1-8.

Haken, H. (1981). Synergetik: Nichtgleichgewichte, phasenubergange und selbstorganisation. Naturwissenschaften, 68, 293-299.

Haken, H., Kelso, J. A. S., \& Bunz, H. (1985). A theoretical model of phase transitions in human hand movements. Biological Cybernetics, 51, 347-356.

Haken, H., Peper, C. E., Beek, P. J., \& Daffertshofer, A. (1996). A model for phase transitions in human hand movements during multifrequency tapping. Physica D: Nonlinear Phenomena, 90, 179-196.

Han, D. W., \& Shea, C. H. (2008). Auditory model: Effects on learning under blocked and random practice schedules. Research Quarterly for Exercise and Sport, 79, 476-486.

Heuer, H., \& Klein, W. (2006). Intermanual interactions related to movement amplitudes and endpoint locations. Journal of Motor Behavior, 38, 126-138.

Hiraga, C. Y., Summers, J. J., \& Temprado, J. J. (2004). Attentional costs of coordinating homologous and non-homologous limbs. Human Movement Science, 23, 415-430.

Hodges, N. J., Chua, R., \& Franks, I. M. (2003). The role of video in facilitating perception and action of a novel coordination movement. Journal of Motor Behavior, 35, 247-260.

Hu, X., \& Newell, K. M. (2011). Visual information gain and task asymmetry interact in bimanual force coordination and control. Experimental Brain Research, 212, 497-504.

Hurley, S. R., \& Lee, T. D. (2006). The influence of augmented feedback and prior learning on the acquisition of a new bimanual coordination pattern. Human Movement Science, 25, 339-348.

Ivry, R., Diedrichsen, J., Spencer, R., Hazeltine, E., \& Semjen, A. (2004). A cognitive Neuroscience perspective on bimanual coordination and interference. In S. P. Swinnen \& J. Duysens (Eds.), Neuro-behavioral determinants of interlimb coordination. Norwell: Kluwer.

Jancke, L., Peters, M., Himmelbach, M., Nosselt, T., Shah, J., \& Steinmetz, H. (2000). fMRI study of bimanual coordination. Neuropsychologia, 38, 164-174.

Kagerer, F. A., Summers, J. J., \& Semjen, A. (2003). Instabilities during antiphase bimanual movements: Are ipsilateral pathways involved? Experimental Brain Research, 151, 489-500.

Kelso, J. A. S. (1981). On the oscillatory nature of movement. Bulletin of the Psychonomics Society, 18, 63.

Kelso, J. A. S. (1984). Phase transitions and critical behavior in human bimanual coordination. American Journal of Physiology:
Regulatory, Integrative, and Comparative Physiology, 15, 10001004.

Kelso, J. A. S. (1995). Dynamic patterns: The self-organization of brain and behavior. Cambridge: The MIT Press.

Kelso, J. A. S., \& deGuzman, G. C. (1988). Order in time: How cooperation between the hands informs the design of the brain. In H. Haken (Ed.), Neural and synergetic computers (pp. 180-196). Berlin: Springer.

Kelso, J. A. S., \& Zanone, P. G. (2002). Coordination dynamics of learning and transfer across different effector systems. Journal of Experimental Psychology: Human Perception and Performance, $28,776-797$

Kelso, J. A. S., Southard, D. L., \& Goodman, D. (1979a). On the coordination of two-handed movements. Journal of Experimental Psychology: Human Perception and Performance, 5, 229-238.

Kelso, J. A. S., Southard, D. L., \& Goodman, D. (1979b). On the nature of interlimb coordination. Science, 204, 1029-1031.

Kelso, J. A. S., Putnam, C. A., \& Goodman, D. (1983). On the space-time structure of human interlimb co-ordination. Quarterly Journal of Experimental Psychology, 35, 347-375.

Kelso, J. A. S., Scholz, J. P., \& SchÖner, G. (1986). Nonequilibrium phase transitions in coordinated biological motion: Critical fluctuations. Physics Letters A, 118, 279-284.

Kelso, J. A. S., DelColle, J. D., \& Schöner, G. (1990). Action-perception as a pattern formation process. In M. Jeanarod (Ed.), Attention and Performance XIII (pp. 139-170). Hillsdale: Erlbaum.

Kelso, J. A. S., Buchanan, J. J., \& Wallace, S. A. (1991). Order parameters for the neural organization of single, multijoint limb movement patterns. Experimental Brain Research, 85, 432-444.

Kelso, J. A. S., Fink, P. W., DeLaplain, C. R., \& Carson, R. G. (2001). Haptic information stabilizes and destabilizes coordination dynamics. Proceedings of the Royal Society of London. Series B: Biological Sciences, 268, 1207-1213.

Kennedy, D., Boyle, J., \& Shea, C. H. (2013a). The role of visual and auditory models in the production bimanual tapping patterns. Experimental Brain Research, 224, 507-518.

Kennedy, D. M., Wang, C., \& Shea, C. H. (2013b). Reacting while moving: Influence of right limb movement on left limb reactions. Experimental Brain Research, 230, 143-152.

Kennedy, D. M., Boyle, J. B., Rhee, J., \& Shea, C. H. (2015a). Rhythmical bimanual force production: Homologous and nonhomologous muscles. Experimental Brain Research, 233, 181-195.

Kennedy, D. M., Boyle, J. B., Wang, C., \& Shea, C. H. (2015b). Bimanual force control: Cooperation or interference. Psychological Research. doi:10.1007/s00426-014-0637-6

Kennerley, S. W., Diedrichsen, J., Hazeltine, E., Semjen, A., \& Ivry, R. B. (2002). Callosotomy patients exhibit temporal uncoupling during continuous bimanual movements. Nature Neuroscience, 5, 376-381.

Kinsbourne, M. (1970). The cerebral basis of lateral asymmetries in attention. Acta Psychologica, 33, 193-201.

Klapp, S. T., Nelson, J. M., \& Jagacinski, R. J. (1998). Can people tap concurrent bimanual rhythms independently. Journal of Motor Behavior, 30, 301-322.

Kovacs, A. J., \& Shea, C. H. (2010). Amplitude differences, spatial assimilation, and integrated feedback in bimanual coordination. Experimental Brain Research, 202, 519-525.

Kovacs, A. J., \& Shea, C. H. (2011). The learning of $90^{\circ}$ continuous relative phase with and without Lissajous feedback: External and internally generated bimanual coordination. Acta Psychologica, 136, 311-320.

Kovacs, A. J., Buchanan, J. J., \& Shea, C. H. (2008). Perceptual influences on Fitt's law. Experimental Brain Research, 190, 99-103.

Kovacs, A. J., Buchanan, J. J., \& Shea, C. H. (2009a). Perception-action coordination dynamics: Using scanning trials to assess coordination tendencies. Neuroscience Letters, 455, 162-167. 
Kovacs, A. J., Buchanan, J. J., \& Shea, C. H. (2009b). Bimanual 1:1 with $90^{\circ}$ continuous phase: Difficult or Easy? Experimental Brain Research, 193, 129-136.

Kovacs, A. J., Buchanan, J. J., \& Shea, C. H. (2010a). Perceptual and attentional influences on continuous 2:1 and 3:2 multi-frequency bimanual coordination. Journal of Experimental Psychology: Human Perception and Performance, 36(4), 936-954.

Kovacs, A. J., Buchanan, J. J., \& Shea, C. H. (2010b). Impossible is nothing: 5:3 and 4:3 multi-frequency bimanual coordination. Experimental Brain Research, 201, 249-259.

Kurtz, S., \& Lee, T. D. (2003). Part and whole perceptual-motor practice of a polyrhythm. Neuroscience Letters, 338, 205-208.

Lai, Q., Shea, C. H., Bruechert, L., \& Little, M. (2002). Auditory model enhances relative timing learning. Journal of Motor Behavior, 34, 299-307.

Lee, T. D., White, M. A., \& Carnahan, H. (1990). On the role of knowledge of results in motor learning: Exploring the guidance hypothesis. Journal of Motor Behavior, 22, 191-208.

Lee, T. D., Swinnen, S. P., \& Verschueren, S. (1995). Relative phase alterations during bimanual skill acquisition. Journal of Motor Behavior, 27(3), 263-274.

Marteniuk, R. G., MacKenzie, C. L., \& Baba, D. M. (1984). Bimanual movement control: Information processing and interaction effects. Quarterly Journal of Experimental Psychology, 36, 335-365.

Maslovat, D., Hodges, N. J., Krigolson, O. E., \& Handy, T. C. (2010). Observational practice benefits are limited to perceptual improvements in the acquisition of a novel coordination skill. Experimental Brain Research, 204, 119-130.

McNevin, N. H., Shea, C. H., \& Wulf, G. (2003). Increasing the distance of an external focus of attention enhances learning. Psychological Research, 67, 22-29.

Mechsner, F., \& Knoblich, G. (2004). Do muscles matter for coordinated action? Journal of Experimental Psychology: Human Perception and Performance, 30, 490-503.

Mechsner, F., Kerzel, D., Knoblich, G., \& Prinz, W. (2001). Perceptual basis of bimanual coordination. Nature, 414, 69-73.

Meesen, R. L. J., Wenderoth, N., Temprado, J. J., Summers, J. J., \& Swinnen, S. P. (2006). The coalition of constraints during coordination of ipsilateral and heterolateral limbs. Experimental Brain Research, 174, 367-375.

Miller, J. (1982). Divided attention evidence for coactivation with redundant signals. Cognitive Psychology, 14, 247-270.

Oullier, O., de Guzman, G. C., Jantzen, K. J., Lagarde, J., \& Kelso, J. A. (2008). Social coordination dynamics: Measuring human bonding. Social Neuroscience, 3, 178-192.

Peper, C. E., Beek, P. J., \& van Wieringen, P. C. W. (1995a). Coupling strength in tapping a 2:3 polyrhythm. Human Movement Science, 14, 217-245.

Peper, C. E., Beek, P. J., \& van Wieringen, P. C. W. (1995b). Multifrequency coordination in bimanual tapping: Asymmetrical coupling and signs of supercriticality. Journal of Experimental Psychology; Human Perception and Performance, 21, 1117-1138.

Peper, C. E., Beek, P. J., \& van Wieringen, P. C. W. (1995c). Frequencyinduced phase-transitions in bimanual tapping. Biological Cybernetics, 73, 301-309.

Peper, C. E., \& Beek, P. J. (1998). Distinguishing between the effects of frequency and amplitude on interlimb coupling in tapping a 2:3 polyrhythm. Experimental Brain Research, 118, 78-92.

Peper, C. E., de Boer, B. J., de Poel, H. J., \& Beek, P. J. (2008). Interlimb coupling strength scales with movement amplitude. Neuroscience Letters, 437, 10-14.

Peters, M. (1989). Do feedback processing, output variability, and spatial complexity account for manual asymmetries. Journal of Motor Behavior, 21, 151-155.

Peters, M. (1994). Does handedness play a role in the coordination of bimanual movements? In S. Swinnen, H. Heuer, J.
Massion, \& P. Casaer (Eds.), Interlimb Coordination: Neural, dynamical, and cognitive constraints (pp. 595-612). San Diego, CA: Academic Press.

Peters, M., \& Schwartz, S. (1989). Coordination of the two hands and effects of attentional manipulation in the production of bimanual 2:3 polyrhythm. Australian Journal of Psychology, 41, 215-224.

Preilowski, B. F. B. (1972). Possible contribution of the anterior forebrain commissures to bilateral motor coordination. Neuropsychlogica, 10, 267-277.

Puttemans, V., Wenderoth, N., \& Swinnen, S. P. (2005). Changes in brain activation during the acquisition of a multifrequency bimanual coordination task: From the cognitive stage to advanced levels of automaticity. The Journal of Neuroscience, $25,4270-4278$.

Ridderikhoff, A., Peper, C. E., \& Beek, P. J. (2005). Unraveling interlimb interactions underlying bimanual coordination. Journal of Neurophysiology, 94, 3112-3125.

Riek, S., Carson, R. G., \& Byblow, W. D. (1992). Spatial and muscular dependencies in bimanual coordination. Journal of Human Movement Sciences, 23, 251-265.

Robertson, I. H., \& Murre, M. J. (1999). Rehabilitation of brain damage: Brain plasticity and principles of guided recovery. Psychological Bulletin, 125, 544-575.

Ryu, Y. U., \& Buchanan, J. J. (2004). Amplitude scaling in a bimanual circle-drawing task: Pattern switching and end-effector variability. Journal of Motor Behavior, 36, 265-279.

Ryu, Y., \& Buchanan, J. J. (2009). Learning an environment-actor coordination skill: Visuomotor transformation and coherency of perceptual structure. Experimental Brain Research, 196, 279-293.

Salesse, R., Temprado, J. J., \& Swinnen, S. P. (2005). Interaction of neuromuscular, spatial and visual contraints on hand-foot coordination dynamics. Human Movement Science, 24, 66-80.

Salmoni, A. W., Schmidt, R. A., \& Walter, C. B. (1984). Knowledge of results and motor learning: A review and critical reappraisal. Psychological Bulletin, 95, 355-386.

Schmidt, R. A. (1991). Frequent augmented feedback can degrade learning: Evidence and interpretations. In J. Requin \& G. E. Stelmach (Eds.), Tutorials in motor neuroscience (pp. 59-75). Dordrecht: Kluwer Academic.

Schmidt, R. A., \& Bjork, R. A. (1992). New conceptualizations of practice: Common principles in three paradigms suggest new concepts for training. Psychological Science, 3, 207-217.

Schmidt, R. A., \& Wulf, G. (1997). Continuous concurrent feedback degrades skill learning: Implication for training and simulation. Human Factors, 39, 509-525.

Schmidt, R. C., Carello, C., \& Turvey, M. T. (1990). Phase transitions and critical Fluctuations in the Visual Coordination of Rhythmic Movement between People. Journal of Experimental Psychology: Human Perception and Performance, 16, 227-247.

Scholz, J. P., \& Kelso, J. A. S. (1989). A quantitative approach to understanding the formation and change of coordinated movement patterns. Journal of Motor Behavior, 21, 122-144.

Scholz, J. P., \& Kelso, J. A. S. (1990). Intentional switching between patterns of bimanual coordination depends on the intrinsic dynamics of the patterns. Journal of Motor Behavior, 22(1), 98-124.

SchÖner, \& Kelso, J. A. S. (1988). Dynamic pattern generation in behavioral and neural systems. Science, 239, 1513-1520.

SchÖner, G., Haken, H., \& Kelso, J. A. S. (1986). A stochastic theory of phase transitions in human hand movement. Biological Cybernetics, $53,247-257$

Scully, D. M., \& Newell, K. M. (1985). Observational learning and the acquisition of motor skills: Toward a visual-perceptual perspective. Journal of Human Movement Studies, 11, 169-186.

Sergent, J. (1993). Music, the brain and ravel. Trends in neuroscience, 16, $168-172$. 
Serrien, D. J., \& Swinnen, S. P. (1997). Isofrequency and multifrequency coordination patterns as a function of the planes of motion. Quarterly Journal of Experimental Psychology Section a - Human Experimental Psychology, 50, 386-404.

Serrien, D. J., Li, Y., Steyvers, M., Debaere, F., \& Swinnen, S. P. (2001). Proprioceptive regulation of interlimb behavior: Interference between passive movement and active coordination dynamics. Experimental Brain Research, 140, 411-419.

Shea, C. H., Wulf, G., Park, J. H., \& Gaunt, B. (2001). Effects of an auditory model on the learning of relative and absolute timing. Journal of Motor Behavior, 33, 127-138.

Shea, C. H., Kovacs, A. J., \& Buchanan, J. J. (2009). Perceptual and Attentional Influences on Bimanual Coordination. In A. Moral (Ed.), Art in Motion: Musical and Athletic Motor Learning and Performance (pp. 227-253). Frankfurt: Peter Lang.

Shea, C. H., Boyles, J., \& Kovacs, A. J. (2012). Bimanual Fitts' tasks: Kelso, Southard, \& Goodman (1979) revisited. Experimental Brain Research, 216, 113-121.

Sherwood, D. E. (1994). Interlimb amplitude differences, spatial assimilations, and the temporal structure of rapid bimanual movements. Human Movement Science, 13, 841-860.

Sigrist, R., Rauter, G., Riener, R., \& Wolf, P. (2013). Augmented visual, auditory, haptic, and multimodal feedback in motor learning: A review. Psychological Bulletin and Review, 20, 21-53.

Sparrow, W. A., \& Summers, J. J. (1992). Performance on trials without knowledge of results $(\mathrm{KR})$ in reduced relative frequency presentations of KR. Journal of Motor Behavior, 24, 197-209.

Spencer, R. M. C., Ivry, R. B., Cattaert, D., \& Semjen, A. (2005). Bimanual coordination during rhythmic movements in the absence of somatosensory feedback. Journal of Neurophysiology, 94, 2901-2910.

Spencer, R. M. C., \& Ivry, R. B. (2007). The temporal represenation of inphase and anti-phase movements. Human Movement Science, 26, 226-234.

Spijkers, W., Tachmatzidis, K., Debus, G., Fischer, M., \& Kausche, I. (1994). Temporal coordination of alternative and simultaneous aiming movements of constrained timing structure. Psychological Research, 57, 20-29.

Stephan, K.M., Binkofski, F., Halsband, U., Dohle, C., Wunderlich, G., Schnitzler, A.,... \& Freund, H.J. (1999). The role of ventral medial wall motor areas in bimanual coordination: A combined lesion and activation study. Brain, 122, 351-368.

Sternad, D., Turvey, M. T., \& Saltzman, E. L. (1999a). Dynamics of 1:2 coordination: Generalizing relative phase to n:m rhythms. Journal of Motor Behavior, 31, 207-223.

Sternad, D., Turvey, M. T., \& Saltzman, E. L. (1999b). Dynamics of 1:2 coordination: Sources of symmetry breaking. Journal of Motor Behavior, 31, 224-235.

Summers, J. J., Todd, J. A., \& Kim, Y. H. (1993). The influence of perceptual and motor factors on bimanual coordination in polyrhythmic tapping task. Psychological Research, 55, 107-125.

Summers, J. J., Davis, A. S., \& Byblow, W. D. (2002). The acquisition of bimanual coordination is mediated by anisotropic coupling between the hands. Human Movement Science, 21, 699-721.

Summers, J. J., Maeder, S., Hiraga, C. Y., \& Alexander, J. R. M. (2008). Coordination dynamics and attentional cost of continuous and discontinuous bimanual circle drawing movements. Human Movement Science, 27, 823-837.

Suzuki, S. I. (1969). Nurtured by love: A new approach to education. New York: Exposition Press.

Swinnen, S. P. (2002). Intermanual coordination: From behavioral principles to neural-network interactions. Nature, 3, 350-361.

Swinnen, S. P., \& Wenderoth, N. (2004). Two hands, one brain: Cognitive neuroscience of bimanual skill. Trends in Cognitive Sciences, 8, 18-25.

Swinnen, S. P., Dounskaia, N., Verschueren, S., Serrien, D. J., \& Daelman, A. (1995). Relative phase destabilization during interlimb coordination: The disruptive role of kinsthetic afferences induced by passive movements. Experimental Brain Research, 3, 439-454.

Swinnen, S. P., Dounskaia, N., Walter, C. B., \& Serrien, D. J. (1997a). Preferred and induced coordination modes during the acquisition of bimanual movements with a 2:1 frequency ratio. Journal of Experimental Psychology: Human Perception and Performance, 23, 1087-1110.

Swinnen, S. P., Lee, T. D., Verschueren, S., Serrien, D. J., \& Bogaerts, D. J. (1997b). Interlimb coordination: Learning and transfer under different feedback conditions. Human Movement Science, 16, 749-785.

Swinnen, S. P., Verchueren, M. P., Bogaerts, H., Dounskaia, N., Lee, T. D., Stelmach, G. E., \& Serrien, D. J. (1998). Age related deficits in motor learning and differences in feedback processing during the production of a bimanual coordination pattern. Cognitive Neuropsychology, 15, 439-466.

Temprado, J. J., \& Swinnen, S. P. (2005). Dynamics of learning and transfer of muscular and spatial relative phase in bimanual coordination: Evidence for abstract directional codes. Experimental Brain Research, 160, 180-188.

Temprado, J. J., Zanone, P. G., Monno, A., \& Laurent, M. (1999). Attentional load associated with performing and stabilizing preferred bimanual patterns. Journal of Experimental Psychology: Human Perception and Performance, 25, 1579-1594.

Temprado, J. J., Swinnen, S. P., Carson, R. G., Tourment, A., \& Laurent, M. (2003). Interaction of directional, neuromuscular and egocentric constraints on the stability of preferred bimanual coordination patterns. Human Movement Science, 22, 339-363.

Treffner, P. J., \& Turvey, M. T. (1993). Resonance constraints on rhythmic movement. Journal of Experimental Psychology: Human Perception and Performance, 19, 339-363.

Treffner, P. J., \& Turvey, M. T. (1995). Handedness and the asymmetric dynamics of bimanual rhythmic coordination. Journal of Experimental Psychology: Human Perception and Performance, 21, 318-333.

Treffner, P. J., \& Turvey, M. T. (1996). Symmetry, broken symmetry and handedness in bimanual coordination dynamics. Experimental Brain Research, 107, 463-478.

Tuller, B., \& Kelso, J. A. S. (1989). Environmentally-specific patterns of movement coordination in normal and split brain subjects. Experimental Brain Research, 75, 306-316.

Wang, C., Kennedy, D. M., Boyle, J., \& Shea, C. H. (2013). A guide to performing difficult bimanual coordination tasks: Just follow the yellow brick road. Experimental Brain Research, 230, 31-40.

Washburn, A., Coey, C. A., Romero, V., \& Richardson, M. J. (2014). Visual multifrequency entrainment: Can 1:2, 2:3 and 3:4 coordination occur spontaneously? Journal of Motor Behavior, 46(4), 247257.

Weeks, D. J., Zelaznik, H., \& Beyak, B. (1993). An empirical note on reduced frequency knowledge of results. Journal of Human Movement Studies, 25, 193-201.

Wilson, A. D., Collins, D. R., \& Bingham, G. P. (2005a). Perceptual coupling in rhythmic movement coordination: Stable perception leads to stable action. Experimental Brain Research, 164, 517-528.

Wilson, A. D., Collins, D. R., \& Bingham, G. P. (2005b). Human movement coordination implicates relative direction as the information for relative phase. Experimental Brain Research, 165, 351-361.

Wilson, A. S., Snapp-Childs, W., Coats, R., \& Bingham, G. P. (2010). Learning a coordinated rhythimical movement with task-appropriate coordination feedback. Experimental Brain Research, 205, 513-520.

Winstein, C. J., \& Schmidt, R. A. (1990). Reduced frequency of knowledge of results enhances motor skill learning. Journal of Experimental Psychology: Learning, Memory and Cognition, 16, 677-691. 
Wulf, G. (2007). Attention and motor skill learning. Champaign: Human Kinetics.

Wulf, G. (2013). Attentional focus and motor learning: A review of 15 years. International Review of Sport and Exercise Psychology, 16, 77-104.

Wulf, G., \& Su, J. (2007). An external focus of attention enhances golf shot accuracy in beginners and experts. Research Quarterly for Exercise and Sport, 78, 384-389.

Wulf, G., Lauterbach, B., \& Toole, T. (1999). Learning advantages of an external focus of attention in golf. Research Quarterly for Exercise and Sport, 70, 120-126.
Yamanishi, J., Kawato, M., \& Suzuki, R. (1980). Two coupled oscillators as a model for the coordinated finger tapping by both hands. Biological Cybernetics, 37, 219-225.

Zaal, F. T. J. M., Bingham, G. P., \& Schmidt, R. C. (2000). Visual perception of mean relative phase and phase variability. Journal of Experimental Psychology: Human Performance and Perception, 26, 1209-1220.

Zanone, P. G., \& Kelso, J. A. S. (1992). Evolution of behavioral attractors with learning: Nonequilibrium phase-transitions. Journal of Experimental Psychology: Human Performance and Perception, 18, 403-421. 